\title{
Bibliographical Materials for Afrikaans Etymological Lexicography
}

Jeremy Bergerson, Department of German, University of California, Berkeley, United States of America (jbergerson@berkeley.edu)

\begin{abstract}
Afrikaans etymological lexicography has yet to see an analytic dictionary along the lines of Sigmund Feist's Gotisches etymologisches Wörterbuch or Anatoly Liberman's An Analytic Dictionary of English Etymology. Before an analytic dictionary of Afrikaans etymology can be written, a comprehensive bibliography of works and articles on the subject must first be brought together. This collection is a first step towards creating a resource for Afrikaans similar to Liberman's $A$ Bibliography of English Etymology.
\end{abstract}

Keywords: ETYMOLOGY, LEXICOGRAPHY, HISTORICAL LEXICOLOGY, DICTIONARIES, WORD STUDIES, BIBLIOGRAPHY, ANALYTIC, AFRIKAANS, HISTORICAL LINGUISTICS

Opsomming: Bibliografiese materiaal vir die Afrikaanse etimologiese leksikografie. Die Afrikaanse etimologiese leksikografie moet nog 'n analitiese woordeboek kry min of meer soos Sigmund Feist se Götisches etymologisches Wörterbuch of Anatoly Liberman se An Analytic Dictionary of English Etymology. Voordat 'n analitiese woordeboek van die Afrikaanse etimologie geskryf kan word, moet 'n omvattende bibliografie van werke en artikels oor die onderwerp eers bymekaargebring word. Hierdie versameling is 'n eerste stap tot die totstandbrenging van 'n hulpmiddel vir Afrikaans soortgelyk aan Liberman se A Bibliography of English Etymology.

Sleutelwoorde: ETIMOLOGIE, LEKSIKOGRAFIE, HISTORIESE LEKSIKOLOGIE, WOORDEBOEKE, WOORDSTUDIE, BIBLIOGRAFIE, ANALITIES, AFRIKAANS, HISTORIESE TAALKUNDE

As etymologies usually appear in dictionaries, one would expect below a discussion of the history of Afrikaans lexicography. The publication of Gouws and Ponelis, ${ }^{1}$ however, has rendered another detailed treatment of the subject unnecessary. Nevertheless, bearing in mind the somewhat obscure development of Afrikaans, the earliest commentaries upon Afrikaans-Hollands, or Cape Dutch, ${ }^{2}$ assume importance as starting points in the history of Afrikaans etymology. Given the scope of this project, moreover, it seems worthwhile to provide some background to the materials handled here.

In the course of the eighteenth century and into the nineteenth, travellers, botanists, and foreigners in the Dutch East India Company's employ remarked upon the local speech at the Cape of Good Hope. Since none of these sojourners 
were trained linguists, they tended not to comment on phonological or otherwise arcane linguistic matters. Instead, they focused on the most noticeable of deviations, word usage. ${ }^{3}$ Their numerous tracts and travelogues are of great value to the etymologist, providing as they do important material for the dating of individual words. J. du P. Scholtz ${ }^{4}$ excerpted all of these texts to write 'Leksikologiese aantekeninge uit ou Kaapse bronne'. It remains the most authoritative study to date.

Starting in the mid 1820s and continuing through the rest of the century one comes across numerous letters and articles written in varieties of Afrikaans and appearing in various newspapers (among others De Zuid-Afrikaan and Het Kaapsche Grensblad). These writings did not go unnoticed, and in Cape publications such as The South African Magazine and The Cape Monthly Magazine, polemical as well as expository articles on 'Cape Dutch' followed that drew heavily on particular words to shore up vying explanations. The participants in these debates were, among others, Pres. F.W. Reitz (then Chief Justice of the Republic of the Free State) and M.L. Wessels (a Cape advocate), and despite their learned tenor and sometimes accurate speculations, these disputations, though they proved influential in spurring on later scholars, are on the whole unscientific. $^{5}$

The earnest study of the Afrikaans lexicon begins with A.N.E. Changuion's 'Proeve van Kaapsch Taaleigen' which appeared as a supplement to the second edition of his Nederduitsche taal in Zuid-Afrika hersteld. It was, by the author's own admission, intended to provide some linguistic value, ${ }^{6}$ but the work is dominated by pedantic caveats. ${ }^{7}$ This and seven other early and particularly useful word lists (spanning the years 1848-1908) were brought together in Van der Merwe (1971). Among these early attempts at describing the Afrikaans vocabulary is Proeve van een Kaapsch-Hollandsch idioticon by N. Mansvelt. His little 'idioticon' contains a goodly amount of information from previous word lists, and provides interesting notes and etymologies of its own. Its effect upon the thinking of later scholars (Hugo Schuchardt, D.C. Hesseling) together with its numerous insights makes it one of the principal texts in Afrikaans etymology. But in spite of all the interesting conjecture and analysis represented in this body of work, none of it ever reached the standard by D.C. Hesseling. 8

The polemic between Hesseling and D.B. Bosman, and tangentially P.J. du Toit, is the first serious debate about the origin of Afrikaans and forms the basis of scholarly literature on the subject. This had great influence on S.P.E. Boshoff, whose dissertation Volk en taal van Suid-Afrika is indispensable to the etymologist. Boshoff went on to author Etimologiese woordeboek van Afrikaans, and later to co-author Afrikaanse etimologieë with G.S. Nienaber. For the rest of the lexicographical-etymological history of Afrikaans, see Gouws and Ponelis.

Deciding which etymologies to include in this bibliography proved difficult. For example, one often comes across a statement like 'baie (< Mal. banjak)'. Here the author has identified the parent language of the word baie, but has failed to provide a discussion. This is not an etymology. Such instances are 
simply dealt with, however, as was the case in two particular works on Afrikaans words, ${ }^{9}$ some etymologies seemed promising, but proved too questionable to enter here. In moments of unsurity, common sense prevailed; e.g. if the author was a main contributor to the field (Abel Coetzee, G.S. Nienaber, J. du P. Scholtz), he was rather included in the bibliography than not. So the number of useless (uninformed, trivial, undeveloped) etymologies has been kept to a minimum.

Deciding which words to enter was easier: the principles behind choosing which words to include in the present bibliography are consistent with those employed in Afrikaanse etimologieë. That is, words shared with Dutch, such as broer, praat, or stoel are left out because they have already been dealt with by Dutch etymologists. However, some words that are clearly Dutch but have a meaning unique to Afrikaans have been included, like die 'the' (Dutch 'those'; relative pronoun), lemoen 'an orange' (Dutch 'lemon') and seun 'boy' (Dutch 'son'). Also, all common Afrikaans words that are dialectal in Dutch are incorporated into the list, e.g. dalk 'maybe,' kiewiet 'a type of bird,' and stadig 'slow.' Some words have enjoyed much attention because they have a phonetic form that is significantly different from Dutch: die 'the,' het 'have,' hom 'him,' hulle 'they, them, their,' watter 'which.' These have also been included. Some words are well-represented (baie, ons), and many others have only one reference. This is due to the fact that the majority of the best etymologies are to be found in books, see the list below. The entry of foreign words has been kept to a minimum, unless they were either words that showed up repeatedly in the literature or words that comprise an integral part of the Afrikaans vocabulary. ${ }^{10}$ The list contains a few obsolete words, such as jaarhonderd 'century,' sulle 'they,' and tof 'smart, dignified.' As a rule, words have been included only when the place of their origin is ascertainable. Since Scholtz supplied important comments regarding the origin or nature of words in Afrikaans, his works have been excerpted even when he did not provide etymologies. Plant and animal names were generally not added, being so numerous as to merit a separate study. Though the scope of this bibliography has been limited to works in Afrikaans, a few important Dutch, English, and German articles have been added, without which the bibliography would have been incomplete. But for a few exceptions, newspaper articles have been omitted.

The definitions in the word list are meant for identification only. Here I followed the format of the Bibliography of English Etymology being prepared at the University of Minnesota. To determine the spelling of the words here, the Afrikaanse woordelys en spelreëls and other authoritative dictionaries such as the Woordeboek van die Afrikaanse Taal and Verklarende Handwoordeboek van die Afrikaanse Taal were consulted.

A few articles about the Afrikaans vocabulary do not contain etymologies but are useful to the etymologist:

Du Plessis-Müller, S.F. 1938-39. Die woordeskat van die Dagboek van Louis Trichardt. TWK 17: 31-9, 56-9 
Du Toit, S.J. 1938. Die Gordon-versameling. TWK 16: 108-11

Muir, John. 1929. Gewone plantname in Riversdal. Huisgenoot 30 Augustus: 45, 47, 49, 51, 55

Paardekooper, P.C. 1990. Hollandse zeemanstaal(?) en Afrikaanse waltaal. TNTL 106: $167-82$

Smuts, J. 1959. Die woordeskat van 'n Voortrekker. TWK (NR) 19(2): 213-17

Smuts, J. 1964. Betekenisontwikkeling in Afrikaans. TVV 20(4): 22-31

Smuts, J. 1969. Leksikologiese aantekeninge by die Kaapse Taalargief. TVV 25(2): 22-6

Van Oordt, L.C. 1978. Verwysings na volkskundige gegewens in die Kaapse argief, met enkele opmerkinge (1692-1823). TVV 34(2): 35-41.

In addition to the above-mentioned works, the two dialect atlases of Afrikaans (Louw 1959, 1975-79) also contain data which are germane to the history of Afrikaans words. In compiling the following bibliography, I made extensive use of Kettley (1970) and Nienaber (1947-68), both of which provided numerous entries of importance to the subject at hand.

The Woordeboek van die Afrikaanse Taal (WAT) is of a hybrid nature, having been initially intended as a synchronic, descriptive dictionary, and under subsequent editors altered to include more or less historical material which can be of interest to the etymologist. As discussed by Botha (1996), the treatment of etymology proper in the WAT has been erratic, though certain volumes contain useful word-historical insights, especially volumes VI-X.

My thanks are due to the Suid-Afrika Huis in Amsterdam for allowing me unrestricted access to their holdings and to Corine de Maier for her great help in locating periodicals, and to the staff of the Special Collections of the J.S. Gericke Library at the University of Stellenbosch, especially Mimi van der Merwe who went to great lengths to locate obscure but necessary articles. Were it not for the Interlibrary Loans at the Wilson Library at the University of Minnesota, there would be no bibliography to publish. I am also grateful to Erika Terblanche at the National Afrikaans Literary Museum and Research Centre in Bloemfontein, Republic of South Africa, as well as Crystal Warren and Malcolm Hacksley from the National English Literary Museum in Grahamstown, Republic of South Africa. Many thanks to Tanja Harteveld from the Bureau of the Woordeboek van die Afrikaanse Taal in Stellenbosch. Finally, without the support of Anatoly Liberman, this project would never have been undertaken and seen through.

Though articles on Afrikaans etymology are indispensable and were in need of being brought together and annotated, the majority of the more luminary word histories are in books. These books, along with a few articles are listed below. They all have word lists or are otherwise easy to use. Hesseling (1899, 1923), Du Toit (1905), Bosman (1916), and Scholtz (1963, 1972) all lack word lists, and, being the seminal works that they are, are included in the main body of the bibliography. 


\section{Notes}

1. Gouws, R.H. and F.A. Ponelis. 1992. The Development of Afrikaans and the Lexicographical Tradition. Zgusta, Ladislav (Ed.) History, Languages, and Lexicographers: 77-104. Tübingen: Niemeyer.

2. Both terms are understood here to indicate a form of Afrikaans that existed from possibly the beginning of the Colony, but certainly by 1775 , to roughly the end of the nineteenth century, that was perceived by its speakers as being Dutch, despite its idiosyncrasies.

3. One famous such instance is Mentzel's oft-quoted observation on the use of ons for wij amongst the womenfolk of the Cape: 'Die Sprache der Landleute ist so wenig reine Holländische Mundart als die teutschen Bauern reines Teutsch sprechen. Die Mannspersonen nehmen das Maul dabei sehr voll, und das Frauenvolk hat Redensarten angenommen, die zuweilen recht lächerlich sind. Zum Exempel. Man frägt etwan, ob sie keine Bibel haben, so erfolgt die Antwort: Onz heeft geen Bijbel ... Wenn man sie aber aldann frägt: Wie viel Unzen gehen auf ein Pfund? so werden die schamroth.' (Vol. II chap. 7) Mentzel, O.F. 1785-87. Vollständige geographische und topographische Beschreibung des afrikanischen Vorgebirges der Guten Hoffnung. Glogau.

4. Scholtz, J. du P. 1965. Afrikaans uit die vroeë tyd: 146-204. Cape Town: Nasou.

5. For a thorough discussion of this literature, see Scholtz, J. du P. 1985. Vroeë taalkundige studies. TGw 25(2): 82-93.

6. 'Heeft het zijne belangerijke zijde, om op te merken, hoe het Nederduitsch van het eene gewest van Nederland van dat van het andere verschilt, en hoe, door vergelijking, het eene taalgebruik het andere toelicht en opheldert, dan kan het ook niet onbelangerijk zijn, de eigenheden van het Kaapsch-Nederduitsch bijéén gesteld te zien.' (Van der Merwe 1972: 5.)

7. 'Het hoofddoel van de volgende verzameling, gelijk men al dadelijk uit den titel van ons werk kan afleiden, was om het Nederduitsch, voor zoo ver de taal, die in deze Kolonie gesproken wordt, dien naam dragen mag, van deels geheel vreemde, deels verminkte woorden en spreekwijzen te zuiveren, of althans den weg daartoe aan te wijzen.' (Van der Merwe 1972: 5.) Luckily his efforts were for nought, as one inhabitant of Montagu states, 'Jij kan ver mij gloo dat die plat Hollans meer gelees wor onder ons boere as die wat Sankion ver ons wil leer in zijn boekies'. Scholtz, J. du P. 1965. Die Afrikaner en sy taal 1806-1875: 180. Cape Town: Nasou.

8. For more on this scholarship, see Loubser, C. De B. 1950. Die studie van die Afrikaanse woordeskat in die 19de eeu. Unpublished M.A. Thesis. Cape Town: University of Cape Town.

9. Given how much space these articles would take up, the fact that only some of the words in each study would merit entry here, and that they are organized like dictionaries, Du Plooy 1981 and Leal 1978 were not excerpted and are in the list below of easily consulted works.

10. For a thorough discussion of the Malay aspect of the Afrikaans vocabulary, see Poole, C.H.. 1951. ' $n$ Kritiese beskouing van die Maleise en Portugese woorde in Afrikaans. Unpublished M.A. Thesis. Cape Town: University of Cape Town. For works on Portuguese words, see Leal (1978) in addition thereto. A further study on Portuguese words in Afrikaans, is that by Rebelo, I.M.F. 1982. Opmerkings oor Portugese woorde in Afrikaans. Unpublished B.A. Hons. Thesis. Johannesburg: University of the Witwatersrand. 


\section{References}

Boshoff, S.P.E. 1921. Volk en taal van Suid-Afrika. Pretoria: J.H. de Bussy.

Boshoff, S.P.E. 1936. Etimologiese woordeboek van Afrikaans. Cape Town: Nasionale Pers.

Boshoff, S.P.E. and G.S. Nienaber. 1967. Afrikaanse etimologieë. Pretoria: Die Suid-Afrikaanse Akademie vir Wetenskap en Kuns.

Bosman, D.B. 1923. Oor die ontstaan van Afrikaans. Amsterdam: Swets \& Zeitlinger.

Botha, Willem. 1996. Die WAT en etimologie: Word die kringloop voltooi? Lexikos 6: 159-170.

Du Plooy, Floris D. 1981. Moontlike raakpunte tussen Platduits en Afrikaans. Wetenskaplike bydraes van die PU vir $\mathrm{CHO}$, Reeks A: Geesteswetenskappe, Nr. 41. Potchefstroom: Potchefstroom University for CHE.

Franken, J.L.M. 1953a. Taalhistoriese bydraes. Amsterdam/Cape Town: A.A. Balkema.

Franken, J.L.M. 1953b. Tentatiewe etimologieë. Annale van die Universiteit van Stellenbosch, Jaargang 27, Reeks B, No. 1. Stellenbosch: University of Stellenbosch.

Hesseling, D.C. 1905. Het Negerhollandsch der Deense Antillen. Leyden: Sijthoff.

Kempen, W. n.d. Woordvorming en funksiewisseling in Afrikaans. Cape Town: Nasionale Boekhandel.

Kempen, W. n.d. Samestelling, afleiding en woordsoortlike meerfunksionaliteit in Afrikaans. Cape Town: Nasou.

Kettley, G.M. (Comp.). 1970. Suid-Afrikaanse linguistiese bronnegids. Raad vir Geesteswetenskaplike Navorsing, Publikasiereeks Nr. 11. Johannesburg: Voortrekkerpers.

Kloeke, G.G. 1950. Herkomst en groei van het Afrikaans. Leyden: Universitaire Pers.

Leal, L. 1978. Die Portugese leksikale elemente in Afrikaans. Wetenskaplike bydraes van die PU vir $\mathrm{CHO}$, Reeks A: Geesteswetenskappe, Nr. 28. Potchefstroom: Potchefstroom University for CHE.

Le Roux, T.H. 1964. Die dagboek van Louis Trigardt. Pretoria: J. L. van Schaik.

Le Roux, T.H. 1968. Raakpunte tussen Afrikaans en die Nederlandse volkstaal. Deel II: Woordvorm en woordbetekenis. TGw 8: 321-356.

Links, Tony. 1989. So praat ons Namakwalanders. Cape Town: Tafelberg.

Louw, S.A. 1959. Afrikaanse taalatlas. Pretoria: University of Pretoria.

Louw, S.A. in collaboration with L.C. Eksteen. 1975-79. Afrikaanse taalatlas. New series 1-5. Pretoria: University of Pretoria.

Malherbe, D.F. 1924. Afrikaanse spreekwoorde en verwante vorme. Bloemfontein: Nasionale Pers.

Mansvelt, N. 1884. Proeve van een Kaapsch-Hollandsch idioticon met toelichtingen en opmerkingen betreffend land, volk en taal. Cape Town: Van de Sandt de Villiers. Also in Van der Merwe 1971: 129222.

Nienaber, G.S. 1963. Hottentots. Pretoria: J.L. van Schaik.

Nienaber, P.J. (Comp.). 1947-68. Bronnegids by die studie van die Afrikaanse taal en letterkunde. Six volumes. Further volumes published by the Raad vir Geesteswetenskaplike Navorsing, Pretoria and the Nasionale Afrikaanse Letterkundige Museum en Navorsingsentrum, Bloemfontein.

Patriot Woordeboek Afrikaans-Engels/Patriot Dictionary Cape Dutch-English. 1902. Paarl: D.F. du Toit. Republished by H.J.J.M. van der Merwe. Pretoria: J.L. van Schaik.

Pettman, Charles. 1913. Africanderisms. London: Longmans, Green and Co. 
Rademeyer, J.H. 1938. Kleurling-Afrikaans. Die taal van die Griekwas en Rehoboth-Basters. Amsterdam: Swets \& Zeitlinger.

Raidt, Edith H. 1994. Historiese taalkunde. Studies oor die geskiedenis van Afrikaans. Johannesburg: Witwatersrand University Press.

Scholtz, J. du P. 1941. Uit die geskiedenis van die naamgewing aan plante en diere in Afrikaans. Cape Town/Bloemfontein/Port Elizabeth: Nasionale Pers.

Scholtz, J. du P. 1965. Afrikaans uit die vroeë tyd: Studies oor die Afrikaanse taal en literêre volkskultuur voor 1875. Cape Town: Nasou.

Scholtz, J. du P. 1972. Boustowwe vir 'n historiese woordeboek van Afrikaans. Afrikaans-Hollands in die agtiende eeu: 106-180. Cape Town: Nasou.

Scholtz, J. du P. 1975. Naamgewing aan plante en diere in Afrikaans: Bydrae tot ' $n$ geskiedenis van die Afrikaanse woordeskat. Second edition. Cape Town: Nasou.

Scholtz, J. du P. 1985. Afrikaanse woorde en uitdrukkinge - eiegoed of erfgoed? TGw 25: 235-90.

Silva, Penny (Mng. Ed.), Wendy Dove, Dorothea Mantzel, Colin Muller, Madeleine Wright. 1996. A Dictionary of South African English on Historical Principles. Oxford/New York: Oxford University Press.

Smith, J.J. 1962. Op ons taalakker. Pretoria: J.L. van Schaik.

Terblanche, H.J. and J.J. Odendaal. 1966. Afrikaanse woordeboek. Verklarend met woordafleidings. Johannesburg: Afrikaanse Pers-Boekhandel.

Van der Merwe, H.J.J.M. 1971. Vroeë Afrikaanse woordelyste. Pretoria: J.L. van Schaik.

Van Oordt, L.C. 1948-1956. Die Kaapse Taalargief. Nrs. 1-4 self-published, 1947-48, nrs. 5-10 in TWK, Apr. 1950-Apr. 1956.

Van Wyk, G.J. (Techn. Ed.), A.E. Cloete, A. Jordaan, H.C. Liebenberg en H.J. Lubbe. 2003. Etimologiewoordeboek van Afrikaans. Stellenbosch: Bureau of the WAT.

Van Wyk, G.J. (Ed.), A.E. Cloete, H.C. Liebenberg, H.J. Lubbe, A. Prinsloo. 2007. Etimologiewoordeboek van Afrikaans. Supplement. Stellenbosch: Bureau of the WAT.

Veth, P.J. 2003. Uit Oost en West. Nicoline van der Sijs (Ed.). Reprint of the 1889 edition. Amsterdam/Antwerpen: Veen.

Von Wielligh, G.R. 1925. Ons geselstaal. 'n Oorsig van gewestelike spraak soos Afrikaans gepraat word. Pretoria: J.L. van Schaik. 


\section{Woordelys / Word List}

aalwyn: 'n plantsoort / aloe, a kind of plant Nienaber 1966, 1968a; Scholtz 1972

aandag: huisgodsdiens / family prayers Hesseling 1899

aapskeloeder: 'n gemene vent / blackguard Hesseling 1899, 1923; Schuchardt 1885

aardig: onaangenaam / unpleasant Bouman 1925-26; Lubbe 2004

abba: ('n kind) op die rug of skouers dra / piggyback, to carry (a child) on the back or shoulders Hesseling 1899

abiekwasgeelhout: 'n houtsoort / tamarisk, a kind of wood Boshoff 1950

afdraand: skuinste / decline Liebenberg 1976

Afrikaner: 'n Afrikaanssprekende persoon / an Afrikaans-speaking person Scholtz 1972

afrokkel: op 'n slinkse manier verkry / to wheedle out of De Villiers 1961

ag: uitroep / oh!, alas!, exclamation Scholtz 1985

agostou: 'n plantsoort / a kind of plant Von Wielligh 1921-22

agterlosig: nalatig / careless Malherbe 1922-23

aia: kinderoppasser / nanny Hesseling 1899

ajoos: 'n swamsoort / puff-ball, a kind of fungus Boshoff 1952

akkerboom: eikeboom / oak tree Scholtz 1985

akkerwanie: 'n grassoort / cuscus, a kind of grass Hesseling 1899

alikreukel: 'n eetbare seeslak / periwinkle, an edible sea slug Smith 1913; Te Winkel 1896

alla: uitroep / (good) gracious, exclamation Bosman 1916; Du Toit 1905; Smith 1913

als: 'n plantsoort / wormwood, a kind of plant Scholtz 1972

amper: byna / almost Bosman 1916; D'Arbez 1916; Hesseling 1899, 1923; Te Winkel 1896; Van Oordt 1916 arrie: uitroep / (I) say!, my!, exclamation Bosman 1916; Hesseling 1899; Schuchardt 1885; Smith 1913

askuus: ekskuus / excuse me Scholtz 1972

aspres: opsetlik / intentionally Scholtz 1972 
assegaai: spies / assegai, speer Bosman 1916; Du Toit 1905; Hesseling 1899, 1923; Te Winkel 1896; Van Oordt 1916

astrak: wisselvorm van ekstrak / variant of ekstrak, extract Scholtz 1972

astrant: parmantig / cheeky, impudent Scholtz 1972

atjar: suurtjies / pickles Bosman 1916; Hesseling 1899

baadjie: 'n bokledingstuk / jacket, an outer garment Bosman 1916; Hesseling 1899; Malherbe 1922-23; Te Winkel 1896

baar: onervare, onbedrewe; ru / inexperienced, unskilled; crude Bosman 1916; Hesseling 1899, 1923; Nienaber 1954i; Schuchardt 1891; Smith 1938; Te Winkel 1896

babbeljoentjie: hemelbed / four-poster, canopy bed Coetzee 1968

bafta: 'n growwe blou materiaal / a coarse blue material Boerevrou; Le Roux 1957

baie: erg, veel, heel / very, many, much, quite Anoniem 1908; Cilliers 1908, 1909; Du Plooy 1966; Hesseling 1899, 1909; Kruyskamp 1946; Scholtz 1963; Smith 1942; Valkhoff 1969; Van Oordt 1916; Von Wielligh 1921-22

bak: kos; 'n houer waarin kos opgedien word / food; a container in which food is served Malherbe 1922-23

baklei: vuisslaan; rusie maak / to have a fist-fight; quarrel Bosman 1916; Hesseling 1899; Scholtz 1972; Te Winkel 1896

balie: 'n oop vat / tub, an open vat Schuchardt 1885

baljaar: luidrugtig speel en rondspring / to play, frolic boisterously Bosman 1916; Hesseling 1897, 1899, 1923; Te Winkel 1896; Valkhoff 1969

basaar: 'n Oosterse markplek / bazaar, an Eastern marketplace Bosman 1916; Hesseling 1899; Van Oordt 1916

basta: genoeg! / enough! Bosman 1916; Hesseling 1899, 1923; Liebenberg 1976; Valkhoff 1969

beesbyter: mamba, 'n soort slang / mamba, a kind of snake Von Wielligh 1921-22

beghaits: befoeterd / cranky Bergerson 2004

beghieks: mal / crazy Bergerson 2004

bejieks: sien beghieks / see beghieks

bejieps: sien beghieks / see beghieks

bejoeks: sien beghieks / see beghieks

bejorries: sien beghieks / see beghieks

betjoeks: sien beghieks / see beghieks betjoins: sien beghieks / see beghieks

betjols: sien beghieks / see beghieks

betjorries: sien beghieks / see beghieks

bermotsersanpeer: ' $n$ peersoort / a kind of pear Hesseling 1899, 1923; Valkhoff 1955 
biebies: luise / lice Vercoullie 1919

biesroei: 'n soort riet / a kind of reed Hesseling 1899, 1923

bietjie: 'n klein hoeveelheid / little, a small quantity Conradie 1994

biltong: repe gedroogde vleis / strips of dried meat Nienaber $1954 \mathrm{n}$

biskop: 'n vissoort / musselcracker, a kind of fish Boshoff 1941

blaar: die plat, skyfvormige deel van 'n plant / leaf, the flat, disc-shaped part of a plant Malherbe 1922-23; Bouman 1922

blaasbalk: 'n werktuig om 'n vuur aan te blaas / bellows, an implement to fan a fire Scholtz 1972

blatjang: 'n dik, gekruide vrugtesous / chutney, a thick spiced fruit sauce Bosman 1937

bleik: laat wit word / to bleach Scholtz 1963

blus: weerstandsvermoë / power of resistance Bouman 1925-26; Malherbe 1922-23

bobotie: gebakte kerriemaalvleis / baked curried mince meat Boerevrou

boeglam: afgemat / exhausted Schuchardt 1885

Boesman: 'n lid van 'n Suid-Afrikaanse stam / Bushman, a member of a South African tribe Den Besten 2004; Lehmann 1945; Nienaber 1952a, 1954f; Van Oordt 1941

boet: broer / brother Bergerson 2002a; Bosman 1916; Du Toit 1905; Hesseling 1923; Vercoullie 1919

bokman: mynwerker / miner De Tollenaere 1957

bokrin: 'n roep om bokke in die kraal te jaag / a call to herd goats into the corral Scholtz 1979; Taalman 1946

bokruit: 'n roep om bokke uit die kraal te jaag / a call to herd goats out of the corral Scholtz 1979; Taalman 1946

bokveld: in die uitdrukking "bokveld toe wees", dood wees / in the expression 'bokveld toe wees', to be dead Lubbe 1967

bolmakiesie: met die voete oor die kop / head over heels Coetzee 1968; Nienaber 1954m

bonkes: 'n kort tabakpypie / a short tobacco pipe Hesseling 1899, 1923

booi: ' $n$ manlike bediende / a male servant Den Besten 2004; Van Oordt 1936

borrie: 'n kruiesoort / turmeric, a kind of spice Hesseling 1899; Smith 1913

botel: 'n plek waar bote gehou word / bo(a)tel, a place where boats are kept Odendal 1964

bottel: 'n glashouer met 'n dun nek / bottle Bosman 1916, 1922-23; Du Toit 1905; Smith 1921-22; Vercoullie 1919 
bra: eintlik; haas / actually; somewhat Conradie 1994

bredie: gestoofde vleis en groente / stew, stewed meat and vegetables Hesseling 1899; Smith 1913

breekspul: deurmekaarspul / mix-up Du Plooy 1966

brei: ('n vel) bewerk om sag en buigsaam te word / to prepare, tan (a skin) to become soft and pliable Scholtz 1963

broesa: die duiwel / the devil Hesseling 1899

burg: 'n gekastreerde varkmannetjie / barrow, hog, a castrated male pig Scholtz 1985

busseltjie: die vulling agter in 'n rok / bustle, the padding in the back of a dress Coetzee 1968

daardie: dié, ding(e) daar / that, those Hesseling 1899; Pauwels 1959a; Roberge 2001; Scholtz 1963, 1972

dalk: miskien, moontlik / maybe, possibly Du Plooy 1966; Scholtz 1963, 1972

dam: 'n oop opgaarplek vir water / dam, an open storage place for water Snijman 1977

die: bepaalde lidwoord / the, definite article Hesseling 1899, 1919, 1923; Kruisinga 1906; Le Roux 1919; Scholtz 1963, 1972; Van den Berg 1965

doepa: toorgoed / magic potion, charms Du Toit 1905

doerias: 'n soort geblomde symateriaal / a kind of floral silk material (vgl./cf. doria) Du Toit 1905

Doesman: Duisman / Dutchman Den Besten 2004

dogter: meisie / girl Du Toit 1905

dolos: kneukelbeen, as kinderspeelgoed en vir waarsêery gebruik / knuckle-bone, used as children's playthings and for soothsaying Boshoff 1951a; Du Toit 1905; Du Toit 1932; Du Toit 1959b; Hesseling 1899; J.B.D. 1932; Lategan 1987; Malherbe 1922-23; Nienaber 1995; Valkhoff 1955

doodgooi: sleggebakte brood / badly baked bread Nienaber 1954k

doodluiters: ongeërg, asof onskuldig / unperturbed, playing innocent Bouman 1928; Du Plooy 1966; Malherbe 1922-23

dop: 'n sopie alkoholiese drank / a tot of alcoholic beverage Du Toit 1958c

dophou: in die oog hou / to watch, keep an eye on Boshoff 1926

Dopper: 'n lid van die Gereformeerde Kerk in Suid-Afrika / a member of the Reformed Church in South Africa Bokhorst 1940; Du Toit 1959c, 1963; H.G.V. 1919

doria: 'n soort kledingstof / a kind of fabric (vgl. / cf. doerias) Boerevrou 
drabok: 'n plantsoort / darnel, a kind of plant Nienaber 1965, 1968a

duiwelsterk: 'n soort kledingstof / a kind of fabric Smith 1921-22

dusketyd: dieselfde tyd / the same time Labuschagne 1936

duwejoe: in die uitdrukking "in die duwejoe wees", onseker wees / in the expression 'in die duwejoe wees', to be unsure Nienaber 1953, 1954c; Scholtz 1972

eena, deena: die eerste woorde van 'n uittelrympie vir kinders / the first words of a children's counting-out rhyme Nienaber 1954a

eendjies: kankerbossie, 'n plantsoort / cancer-bush, a kind of plant Von Wielligh 1921-22

eenspaaierig: nie lief vir geselskap nie / solitary, not fond of company Coetzee 1954

els: 'n priemvormige werktuig vir die maak van gaatjies / alder, an awl-shaped instrument for making small holes Scholtz 1972

emma koelemma: die eerste reël van 'n liedjie / the first line of a song Anoniem 1962; Coetzee 1961

es: vuurherd / fireplace Coetzee 1964

ewwa-trewwa: 'n plantsoort / a kind of plant Malherbe 1922-23; Vercoullie 1919

fieterjasies: tierlantyntjies / fripperies, knick-knacks Nienaber 1994a

filekommis: in die uitdrukking "iemand filekommis maak", onterf / in the expression 'iemand filekommis maak', to disinherit Smith 1921-22

fluit: 'n entmetode van plante / a grafting method of plants Coetzee 1968

froetang: 'n plantsoort / a kind of plant Du Toit 1905; Odendal 1966

gansies: kankerbossie, 'n plantsoort / cancer-bush, a kind of plant Von Wielligh 1921-22

Gatjieponner: 'n spotnaam deur Doppers aan lidmate van ander Afrikaanse kerke gegee / nickname given by Doppers to members of other Afrikaans churches Du Toit 1959c

gebodder: die handeling van kwel, pla, lastig wees / the act of vexing, annoying, being troublesome Du Plooy 1966

geilsiekte: 'n veesiekte veroorsaak deur blousuurvergiftiging/ a livestock disease caused by prussic acid poisoning Smith 1913

geit: meisie / girl

Malherbe 1922-23

geitjie: 'n kleinerige akkedis met gladde skubbe / gecko, a smallish lizard with smooth scales

Boshoff 1927-28; Du Toit 1905; Smith 1921-22 
gevreet: gesig / mug, face

Hesseling 1923

ghantang: vryer; beminde / suitor; sweetheart

Du Toit 1958a

ghoen: 'n groot skietalbaster / taw, a big shooting marble Hesseling 1899, 1923; Smith 1921-22

ghwarrie: gruisgat / quarry, gravel pit Coetzee 1968; Venter 1968

glad: inderdaad / indeed, quite Conradie 1994

goël: deur toertjies bedrieg / to conjure, trick by sleight of hand Scholtz 1963

goiing: grofgeweefde materiaal, dikwels gebruik vir die maak van sakke / hessian, coarsely woven material, often used for making sacks Bosman 1916; Du Toit 1905; Hesseling 1923; Van Oordt 1916

graatjie: stokstertmeerkat, 'n diersoort / suricate, a kind of animal Boshoff 1927-28; Nienaber 1954d

gramadoelas: 'n afgeleë, moeilik bereikbare gebied / outback, a remote, difficultly accessible area

Boshoff 1923-24; Smith 1921-22

gunter: doer, ginds / over there, yonder Scholtz 1972

gus: onvrugbaar, steriel / barren, sterile Smith 1913; Venter 1968

haai: uitroep / hullo!, hey!, exclamation Bosman 1916; Du Toit 1905

haai-hoei: bohaai / ado, hullabaloo Du Toit 1905

haasbek: 'n mond waarin veral die boonste voortande ontbreek / a mouth in which especially the upper front teeth are missing De Tollenaere 1957

hake-krukke: 'n seunspeletjie / a boy's game Du Plooy 1966

hakskeen: die agterste deel van die voet / heel, the back part of the foot Snijman 1977

hakskeenpleister: waardelose papiergeld veral van die vroeëre Zuid-Afrikaansche Republiek / worthless paper money especially from the former Zuid-Afrikaansche Republiek Boerevrou

hanepoot: 'n druifsoort / a variety of grape Hesseling 1916a

harslag: ingewande in die borskas van 'n geslagte dier / pluck, entrails in the thorax of a slaughtered animal Smith 1913

hartbeeshuis: paal-en-klei-huis / wattle and daub hut Nienaber 1952a, 1952b, 1954e, 1989; Walton 1987

Hasie Aboel: 'n kinderspeletjie / catch the hare, a children's game Kloppers 1951-52, 1952-53a, 1952-53b, 1953

hê: infinitiefvorm van het / infinitive form of het Scholtz 1972 
hekel: handwerk vervaardig met gare en 'n haakpen / to crochet Scholtz 1963

herneuters: herneutermes, 'n soort jagmes / Hernnhuter, a kind of hunting-knife Von Wielligh 1921-22

het: verboë vorm van $h \hat{e}$ / conjugated form of $h \hat{e}$ Scholtz 1972, 1985

hierdie: $\operatorname{ding}(\mathrm{e})$ hier / this, these Hesseling 1899; Pauwels 1959a; Roberge 2001; Scholtz 1963, 1972

hoeka: lank gelede / long ago Conradie 1994; Hesseling 1899

hom: persoonlike voornaamwoord, voorwerpsvorm, derde persoon enkelvoud, manlik / him, personal pronoun, object form, third person singular, masculine Scholtz 1963, 1972

Horak: in die uitdrukking "Wat sê Horak?", retoriese vraag om verbasing of twyfel uit te druk / in the expression 'Wat sê Horak?', rhetorical question to express surprize or doubt Du Toit 1959a; Nienaber 1968b

hortjie: 'n raamwerk as bedekking vir 'n deur of venster / louvre, shutter, a covering for a door or window Lubbe 1970

hotom: linksom / to the left Boerevrou

Hottentot: 'n lid van 'n Suid-Afrikaanse stam / a member of a South African tribe Nienaber 1954g, 1956a, 1956b

hulle: persoonlike voornaamwoord, derde persoon meervoud / they, personal pronoun, third person plural Goossens 1994; Scholtz 1963; Vor der Hake 1911

inspan: in die uitdrukking "jong osse/kalwers inspan", vomeer / in the expression 'jong osse/kalwers inspan', to vomit Boshoff 1926

jaarhonderd: eeu / century Hesseling 1899

jamboes: 'n boomsoort / rose apple, a kind of tree Du Toit 1905

japsnoet: 'n vrypostige kind / whipper-snapper, an impertinent child Du Plooy 1966

jilletjie: grap / joke Hesseling 1914

josie: die duiwel / the devil Boshoff 1952; Bosman 1916; Hesseling 1899; Van Oordt 1916

julle: persoonlike voornaamwoord, tweede persoon meervoud / you, personal pronoun, second person plural Goossens 1994; Scholtz 1963

kaaiman: 'n krokodilagtige reptiel / caiman, a crocodilian reptile Bosman 1916; Du Toit 1905

kabaai: ' $n$ los japon / a loose gown Bosman 1916; Coetzee 1933; Hesseling 1899

kaboedel: 'n klomp / caboodle, a lot, pack Bouman 1951; Coetzee 1995 
kaduks: siekerig / unwell Coetzee 1995

kafferkoring: sorghum / sorghum Boshoff 1941; Laubscher 1941

kafoefel: vry / to canoodle Coetzee 1995

kaiings: oorblyfsels wanneer stukkies dierevet uitgebraai word / greaves, remains after bits of animal fat have been rendered Bosman 1937; Scholtz 1963

kalant: in die uitdrukking "'n ou kalant, lank in die land", 'n ervare, uitgeslape persoon / in the expression ' 'n ou kalant, lank in die land', an experienced, shrewd person Bouman 1925-26

kalfater: 'n skip herstel / caulk, to repair a ship Coetzee 1968

kalkoen: 'n pluimveesoort / turkey, a kind of poultry Nienaber 19541

kalossies: 'n blomsoort / ixia, a kind of flower Smith 1913

kamas: 'n leerbedekking vir die kuite / legging, gaiter, a leather covering for the calves Hesseling 1899, 1923

kamma: kwansuis / ostensibly, supposedly Hesseling 1899

kankerbossie: 'n plantsoort / cancer-bush, a kind of plant Von Wielligh 1921-22

kannie-koenie: jaloers / jealous Hesseling 1916a; Pienaar 1928-29

kaparring: 'n Maleise houtsandaal / a Malay wooden sandal Smith 1913

kapater: 'n gekastreerde bokram / a castrated he-goat Bosman 1916; Hesseling 1899, 1914; Smith 1913; Van Oordt 1916

kapel: kobra, 'n slangsoort / cobra, a kind of snake (vgl./cf. pelloe) Smith 1921-22

kapok: sneeu / snow Du Toit 1905

karba: 'n kan vir vloeistowwe / carboy, a container for liquids Hesseling 1899

karbonkel: 'n edelgesteente / garnet, a precious stone Coetzee 1995

kardoes: papiersak / paper bag Bosman 1916; Hesseling 1899; Smith 1913

Kardoes: in die uitdrukking "bende van Kardoes", 'n rumoerige groep / in the expression 'bende van Kardoes', a rowdy group Boerevrou

karet: 'n bagasierak agterop 'n kar of wa / a luggage carrier at the back of a car or wagon

Odendal 1964; Scholtz 1963, 1985

karjol: plesier, pret maak / to make merry, have fun Coetzee 1995 
karkatjie: 'n sweertjie aan die ooglid / sty(e), a pustule on the eyelid De Villiers 1969

karos: 'n kombers van diervel / a blanket of animal skin Hesseling 1899

kasarm: 'n reeks skakelhuisies, 'n vervalle gebou / a row of semidetached houses, a ramshackle building Scholtz 1963

kastig: kwansuis / ostensibly, supposedly Hesseling 1899

kastrol: 'n komvormige pot / saucepan Valkhoff 1955

katel: ledekant / bedstead Bosman 1916; Hesseling 1899; Liebenberg 1976; Schuchardt 1885, 1891; Van Oordt 1916

katjiepiering: 'n blomsoort / gardenia, a kind of flower Vercoullie 1919

katools: jags, wulps / ruttish, horny Du Plooy 1966

kattejag: in die uitdrukking "op die kattejag uit wees", op 'n vrytog uit wees / in the expression 'op die kattejag uit wees', to be out courting Du Plooy 1966

keil: 'n hoë hoed met 'n silindervormige bol / top hat De Tollenaere 1957; Hesseling 1923

kekkelbek: babbelbek, skinderbek / chatterbox, gossip Du Plooy 1966

kennetjie: 'n seunspeletjie / a boy's game Du Toit 1962

keps: pet / cap Bergerson 2004

kerbollie: baadjie / jacket Boerevrou

kerjakker: baljaar / to frolic Coetzee 1995; Du Plooy 1966

kerjoel: deurmekaar beweeg / to swarm, teem Coetzee 1995

kês: (gekookte) dikmelk / (boiled) sour milk Scholtz 1963

kiaat: 'n houtsoort / Cape teak, a kind of wood Boshoff 1951b; Hartwig 1973; Smith 1913

kiepersol: 'n boomsoort/ umbrella-tree, a kind of tree Bosman 1916; De Bruin 1978; Du Toit 1905; Hesseling 1923

kierang: kul by speletjies / to cheat at games Odendal 1966; Te Winkel 1896

kiets: gelykop / quits Smith 1921-22

kiewiet: 'n voëlsoort / plover, peewit, a kind of bird Scholtz 1972

klapper: kokosneut / coconut Bosman 1916; Du Toit 1905

klip: 'n stuk(kie) van 'n gesteente, rots / stone, rock Hesseling 1899 
kluitjie: in die uitdrukking "kluitjies bak", leuens vertel / in the expression 'kluitjies bak', to tell untruths Lubbe 1968

koedoe: 'n soort wildsbok / kudu (koodoo), a kind of antelope Bosman 1916; Du Toit 1905; Smith 1913; Van Oordt 1916

koeël: 'n bol- of silindervormige projektiel om uit 'n vuurwapen af te skiet / bullet Scholtz 1963

koejawel: 'n vrugtesoort/ guava, a kind of fruit Bosman 1916; Du Toit 1905; Scholtz 1972; Smith 1913

Koelie: Indiër / Coolie, Indian Bosman 1916

koerang: sien kierang / see kierang

koernappers: groot, lomp voete / big, clumsy feet Coetzee 1968

koert: hof / court Bosman 1916; Hesseling 1899, 1923

koggelman: 'n soort akkedis / agama, a kind of lizard Smith 1913

kombers: 'n warm bedekking om onder te slaap / blanket Liebenberg 1976

kondee: bolla / chignon Hesseling 1899

kool: in die uitdrukking "by my kool (kolie) waar", dit is werklik waar / in the expression 'by my kool (kolie) waar', it is really true Du Toit 1905

kraal: 'n omheinde ruimte vir vee / corral Bosman 1916; Hesseling 1899

krans: 'n regaf, steil rots / cliff Odendal 1994

kras: liggaamlik en geestelik flink / robust Te Winkel 1896

kremetart: verpoeierde wynsteen / cream of tartar Hesseling 1914

Kretie: in die uitdrukking "Kretie en Pletie", gepeupel / in the expression 'Kretie en Pletie', rabble Hesseling 1899, 1923

kriek: 'n soort insek / cricket, a kind of insect Bosman 1916; Du Toit 1905

kuier: besoek aflê / to visit Van Ginneken 1927

laai: gewoonte / habit Hesseling 1897, 1899

laksel: vermicelli / vermicelli Hesseling 1899

laksman: 'n voëlsoort / fiscal shrike, a kind of bird Hesseling 1899

lawaai: 'n harde geraas / a loud noise Scholtz 1963

lat: wisselvorm van dat / variant of dat, that Leys 2005; Paardekooper 1990 
lekseltjie: 'n klein bietjie / a small quantity

Du Plooy 1966

lemoen: 'n vrugtesoort / orange, a kind of fruit Bosman 1916; Hesseling 1899; Smith 1921-22

lepaai: kombers / blanket Nienaber 1954j

liefêr: geneig / inclined Le Roux 1957

lieplapper: niksnuts / good-for-nothing Du Toit 1905

likkewaan: 'n akkedisagtige reptielsoort / leguan, a kind of lacertine reptile Scholtz 1972

loesing: ' $\mathrm{n}$ pak slae / spanking Hesseling 1923; Van Oordt 1936

lukwart: 'n vrugtesoort / loquat, a kind of fruit Bosman 1916; Hesseling 1899

lumier: daeraad, oggendskemering / dawn, daybreak Valkhoff 1955

lyfstuk: 'n stuk wat by voorkeur gespeel of gesing word / a favorite song to play or sing Franken 1938

ma: moeder / mother, mum Bosman 1916; Hesseling 1899, 1923

maai: moeder / mother Hesseling 1897, 1899

maaidekaai: 'n baasspelerige persoon / a bossy person Boerevrou

maaier: die larwe van 'n vlieg of brommer / maggot Scholtz 1963

maaifoedie: derduiwel, skelm / blackguard, scoundrel Coetzee 1961

makoppa: mamba, 'n slangsoort / mamba, a kind of snake Von Wielligh 1921-22

makou: 'n pluimveesoort / Muscovy duck, a kind of poultry Hesseling 1899, 1923; Smith 1913

makrappa: 'n Swart arbeider / a Black worker Nienaber 1994b, 1995

makrol: amandelkoekie / macaroon Hesseling 1899, 1923

maltrap: 'n ligsinnige, onnadenkende persoon / madcap Du Toit 1958c

mamparra: 'n onverfynde persoon / an uncouth person Nienaber 1954h, 1995

mandoeka: padda / frog Franken 1954

mandoor: toesighouer, opsigter / supervisor, overseer Bosman 1916

manewales: kaskenades, kaperjolle / antics, capers Labuschagne 1936; Scholtz 1972

manel: 'n lang, swart bokledingstuk vir mans / frock-coat Hesseling 1914; Smith 1913 
mannewaar: 'n groot geraas maak / to make a loud noise Boerevrou

marmotjie: 'n diersoort / guinea-pig, a kind of animal Coetzee 1965

maskie: miskien; nieteenstaande / perhaps; nevertheless Bosman 1916; Hesseling 1899, 1923; Meyer 1900; Roberge 2002; Schuchardt 1885; Van Oordt 1936

meestergoed: medisyne / medicine Smith 1921-22

menteneer: beheer; behartig / to control; manage Waher 2001

mielie: 'n graansoort / maize Bosman 1916; Hesseling 1899; Smith 1913; Te Winkel 1896

moeg: uitgeput / tired, fatigued Scholtz 1963

moesoek: meester, meerdere / master, superior Du Toit 1905; Hesseling 1899, 1923

moggel: 'n soort varswatervis / a kind of freshwater fish Scholtz 1963

molskyn: molvel / moleskin Boerevrou

mombakkies: 'n masker / a mask Smith 1913

mos: immers, inderdaad / after all, indeed Bergerson 2002b; Conradie 1995; Roberge 2002; Scholtz 1972

motjie: 'n (getroude) Moesliemvrou / a (married) Muslim woman Scholtz 1963

moveer: lastig val, pla / to pester, trouble Bosman 1916; Hesseling 1899, 1923; Smith 1913; Valkhoff 1955: Van Oordt 1916

nartjie: 'n vrugtesoort / tangerine, mandarin, a kind of fruit Franken 1927; Hesseling 1899

nè: is dit nie so nie? / isn't that so? Venter 1968

nenna: kinderoppasser / nanny Boerevrou; Hesseling 1899, 1923

nimlik: einste / very same Scholtz 1979

nôi: meisie / girl Hesseling 1899, 1923; Scholtz 1963; Schuchardt 1891; Smith 1913, 1921-22; Valkhoff 1969 nonna: jong meesteres / young mistress Du Toit 1905

nooi: sien nôi / see nôi

norring: 'n groot aantal / a mighty lot Boerevrou

nuut: pas of onlangs vervaardig of bekom / new Scholtz 1963

oes: siek; sleg / sick; bad

Pauwels 1959b

oes: ryp gewasse insamel / to harvest Snijman 1977 
oesbeentjie: dolos / knuckle-bone

Van Blerk 1958

oggend: môre / morning

Scholtz 1963

olienhout: 'n houtsoort / wild-olive, a kind of wood Scholtz 1972

ongans: siek / sick

Schoonees 1950

ons: persoonlike voornaamwoord, onderwerpvorm, eerste persoon meervoud / we, personal pronoun, subject form, first person plural

Bosman 1916; Bouman 1924-25; Goossens 1994; Hesseling 1899, 1916a, 1916b, 1922b, 1923; Kruisinga 1906; Malherbe 1924-25; Meyer 1900; Paardekooper 1969; Scholtz 1963; Schuchardt 1885; Schuhmacher 1973; Smith 1913; Van Oordt 1936; Van Rensburg 1996; Vor der Hake 1911

ontwil: in die uitdrukkings "om ... (se) ontwil", "om die ontwil van ...", ter wille van / in the expressions 'om ... (se) ontwil', 'om die ontwil van ...', for the sake of Scholtz 1972

oond: 'n geslote ruimte om in te bak / oven Scholtz 1972

oop: nie toe nie / open, not closed Scholtz 1972

oorkrabbetjie: oorbel / earring Hesseling 1899; Schuchardt 1891

oorlams: taamlik beskaafd; ervare of bedrewe / reasonably civilized; experienced or skilled

Bosman 1916; Hesseling 1899, 1923; Nienaber 1954i; Schuchardt 1891; Smith 1938; Te Winkel 1896; Van Oordt 1916

otjie: vark / pig

De Tollenaere 1957

ou: kêrel / fellow, guy

Botha 1976; Le Roux 1936; P. 1911

ousanna: vuursteengeweer, pangeweer / flintlock (musket), matchlock (musket) Hesseling 1923; Vercoullie 1919

pa: vader / dad, father Bosman 1916; Hesseling 1899, 1923

paai: aanspreekvorm vir 'n ouer man / form of address for an older man Bosman 1916; Hesseling 1897, 1899, 1923; Liebenberg 1976

paaiboelie: iemand met wie kinders bang gemaak word / bogey(man), somebody with whom children are threatened Du Toit 1905; Smith 1921-22

paljas: toorgoed / magic objects Du Toit 1905

pamperlang: flikflooi / to cajole, wheedle De Tollenaere 1957; Malherbe 1922-23

papelellekoors: 'n voorgewende siekte / a feigned illness Hesseling 1923

papkuil: 'n plantsoort / bulrush, a kind of plant Smith 1921-22; Von Wielligh 1921-22

pasella: present / as a present Smith 1921-22 
pawieperske: 'n vrugtesoort / white clingstone peach, a kind of fruit Hesseling 1899, 1923

pelloe: kobra, 'n slangsoort / cobra, a kind of snake (vgl./cf. kapel) Malherbe 1922-23

perske: 'n vrugtesoort / peach, a kind of fruit Smith 1921-22; Von Wielligh 1921-22

piekel: met moeite dra / to lug, drag Du Toit 1905

pienang: 'n gereg van gekruide gestoofde vleis / a dish of spiced stewed meat Du Toit 1905

piering: 'n bordjie waarin 'n koppie staan / saucer Bosman 1916; Valkhoff 1969; Van Oordt 1916

pikkenien: 'n jong Swart seun / piccanin, a young Black boy Hesseling 1899, 1914, 1923; Smith 1921-22; Valkhoff 1955, 1969

pikkewyn: 'n voëlsoort / penguin, a kind of bird Scholtz 1972

platanna: 'n paddasoort / clawed toad, a kind of frog Smith 1913

poelpetaat: tarentaal / guinea-fowl Coetzee 1969

poerbasledan: tot tydverdryf / for passing the time Hesseling 1899; Schuchardt 1885

pondok: hut / hut, shack Bosman 1916; Hesseling 1899; Van Oordt 1916

portfesiedeur: 'n dubbele deur wat kan terugvou / a double door which can fold back Scholtz 1963; Smith 1913

potjierol: vetsak / pudge, fatty De Tollenaere 1957

prinjeel: wisselvorm van prieel / variant of prieel, pergola Scholtz 1972

rabbedoe: 'n wilde, uitgelate kind; 'n seunsagtige meisie / a boisterous, uninhibited child; a tomboy Bosman 1916; Hesseling 1899, 1923; Schuchardt 1891; Van der Meulen 1917

ramkie: 'n eenvoudige soort kitaar / a simple kind of guitar Scholtz 1963; Smith 1913, 1921-22

ransel: 'n pak slae gee / to spank, flog Lubbe 1968

rappelkops: duiselig / dizzy Hesseling 1899

rasper: gereedskap met tandagtige uitsteeksels / rasp, grater, utensil with toothlike extensions Scholtz 1972

ratel: 'n diersoort / badger, a kind of animal Boshoff 1941

rêrig: werklik / really Nienaber 1991; Scholtz 1963

resep: voorskrif vir die bereiding van kos of drank / recipe Scholtz 1963

resnawel: redelik, werklik / reasonably, really Nienaber 1968a; Waher 2001 
rinneweer: verniel / to ruin, damage Scholtz 1972

rissie: 'n peul van 'n soort speseryplant / chilli, the pod of a kind of spice plant Bosman 1916; Hesseling 1899; Smith 1913; Van Oordt 1916

rondawel: ' $n$ ronde hut / a round hut Du Toit 1926-27; Hesseling 1914; Nienaber 1968a

rooms: struikelend (ten opsigte van 'n perd) / faltering (with regard to a horse) De Tollenaere 1957

rottang: 'n plantsoort / rattan, cane, a kind of plant Bosman 1937; Scholtz 1963, 1972

rysmier: 'n soort insek / termite, a kind of insect Nienaber 1954b

sa: uitroep om ' $n$ hond aan te hits / hist!, exclamation to set a dog on Smith 1913; Van Oordt 1936

sambok: 'n swaar sweep / sjambok, a heavy whip Hesseling 1899; Te Winkel 1896

sambreel: 'n opvoubare son- of reënskerm / umbrella Bouman 1922; Hesseling 1899; Te Winkel 1896

sammajoa: om 't ewe / all the same Du Toit 1905

sarlot: 'n soort ui / shallot, a kind of onion Scholtz 1972

se: besitlike partikel / possessive particle Schuchardt 1885; Scholtz 1963

seekoei: 'n diersoort / hippopotamus, a kind of animal Hesseling 1914; Smith 1913, 1921-22; Von Wielligh 1921-22

sens: 'n sekelvormige werktuig om graan mee te maai / scythe Scholtz 1963, 1972

seroet: sigaar / cheroot, cigar Hesseling 1899

seties: 'n soort dans / scottische, a kind of dance Malherbe 1922-23

seun: 'n kind van die manlike geslag / boy Bosman 1916; Hesseling 1899, 1923

sieal: wat teenspoed veroorsaak / which causes misfortune De Villiers 1974; Malherbe 1922-23

sielverkoper: 'n persoon wat matrose onder valse voorwendsels werf / soul-trader, a person who recruits sailors under false pretexts Hesseling 1916a

siembamba: in die liedjie "Siembamba, mama se kindjie" / in the song 'Siembamba, mama se kindjie' Alberts 1955, 1961, 1989; Boshoff 1917

sies: uitroep van walging / bah, exclamation of disgust Bosman 1916; Du Toit 1905

skaai: vaslê, steel / to pinch, steal Smith 1913

skaaprin: 'n roep om skape in die kraal te jaag / a call to herd sheep into the corral Taalman 1946

skaapruit: 'n roep om skape uit die kraal te jaag / a call to herd sheep out of the corral Taalman 1946 
skalk: skelm / rogue, rascal

Lubbe 1970

skalt en walt: rondslenter / to saunter, idle about

Malherbe 1922-23

skinder: agteraf kwaadpraat / to slander, gossip Du Plooy 1966; Scholtz 1972

skoot: keer, maal / time Du Plooy 1966

smous: 'n rondreisende handelaar / pedlar, an itinerant trader Meyer 1900

snaaks: vreemd / weird Coetzee 1968

soebat: smeek / to plead Bosman 1916; Hesseling 1899, 1916b, 1923; Te Winkel 1896; Van Oordt 1916

soms: somtyds; af en toe / sometimes; now and then Scholtz 1963

sooibrand: pirosis / heartburn, pyrosis Scholtz 1963

sosatie: blokkies gekruide vleis aan 'n pen gaargemaak / kabob, cubes of spiced meat cooked on a skewer Du Toit 1905

spandabel: verkwistend / wasteful, extravagent Waher 2001

spens: 'n vertrek waar voedsel gebêre word / pantry Bosman 1916; Hesseling 1897, 1899, 1914; Liebenberg 1976

spons: sponning / bung

Bergerson 2004

sproei/spru: ontsteking van die mondslymvlies / thrush, sprue Scholtz 1963

stapelgek: heeltemal gek, mal / stark, raving mad Lubbe 1968

stawel: wisselvorm van stapel / variant of stapel, stack Scholtz 1972

stols: trots / proud Hesseling 1899

stoof: 'n toestel vir kook of verwarming / stove Bouman 1922

strandjut: 'n diersoort / beach hyena, a kind of animal Coetzee 1944

strawasie: moeilikheid / difficulty Hesseling 1923

streepsuiker: 'n afranseling / a whooping Malherbe 1922-23

stryk: in die uitdrukking "van stryk af", in die war, verbouereerd / in the expression 'van stryk af', in a tangle, flustered Lubbe 1970

sulle: persoonlike voornaamwoord, derde persoon meervoud / they, personal pronoun, third person plural Scholtz 1963 
swael: 'n voëlsoort / swallow, a kind of bird

Scholtz 1972

swernoot: skurk / rascal

Hesseling 1899; Smith 1913

swets: vloek / to curse

Lubbe 1968

swetterjoel: menigte, klomp / swarm, multitude Scholtz 1972

ta: pa / dad Bosman 1916; Du Toit 1905

tabeetjies: presentjies / small presents Hesseling 1899, 1923

talie: in die uitdrukking "rondomtalie draai" / in the expression 'rondomtalie draai', turn round and round Bosman 1916; Hesseling 1899, 1923; Liebenberg 1976

tamaai: enorm / enormous

Hesseling 1899; Scholtz 1963; Smith 1913, 1921-22; Valkhoff 1969

tamboekie: Temboe / Tembu Scholtz 1972

tameletjie: 'n soort lekkergoed / a kind of candy Scholtz 1972

tarentaal: 'n voëlsoort / guinea fowl, a kind of bird Coetzee 1969; Nienaber 19541; Scholtz 1972; Smith 1921-22

tasal: repies gesoute en gekruide vleis wat winddroog gemaak is / strips of salted and spiced meat which have been air-dried Du Toit 1905; Smith 1913

teak: djatihout, 'n houtsoort / teak, a kind of wood Boshoff 1951b; Hartwig 1973

teiken: skyf; doelwit / target; goal Scholtz 1963

ter duiwel: uitroep, vloekwoord / the deuce!, exclamation, swear word Hesseling 1899

tet: vrouebors / breast Du Toit 1905

tiekie: trippens / tickey, three pence Jeffreys 1952; Smith 1921-22

tier: luiperd, 'n diersoort / leopard, a kind of animal Bouman 1928; Hesseling 1899

tjalie: sjaal / shawl Du Toit 1905

tjap: stempel / stamp Bosman 1916; Hesseling 1899

tjôwa: probeer / to try Scholtz 1972

toering: 'n Maleise strooihoed / a Malay straw hat Du Toit 1905

tof: deftig, waardig / smart, dignified Rautenbach 1978

toiings: flenters, pluiings / rags, tatters Bosman 1937; Malherbe 1922-23; Scholtz 1963 
trassie: 'n onvrugbare dier / a barren animal Bosman 1916; Du Toit 1905

trawaat: ' $\mathrm{n}$ sterk wind / a strong wind Hesseling 1899, 1923

trawal: moeilikheid / trouble Hesseling 1897, 1899

tronk: gevangenis / prison Hesseling 1897, 1899, 1922a, 1923; Valkhoff 1955, 1969

twak: tabak / tobacco Scholtz 1972

tweealfaamsvat: ' $n$ soort vat / a kind of vat Le Roux 1957

u: persoonlike voornaamwoord, tweede persoon hoflik / personal pronoun, second person polite Scholtz 1963; Vor der Hake 1911

uitwiks: 'n pak slae gee / to spank Hesseling 1899

vaderlands: met betrekking tot Nederland / referring to the Netherlands Snijman 1977

vark: 'n diersoort / pig, a kind of animal Scholtz 1972

veels geluk: baie geluk / congratulations Hesseling 1899; Schuchardt 1885

vergange: vervloë; onlangs / bygone; recently Hesseling 1899

vergeet-my-nietjie: 'n blomsoort / forget-me-not, a kind of flower Anoniem 1921

vieruurtjie: 'n blomsoort / afternoon-lady, a kind of flower Bosman 1916; Du Toit 1905; Franken 1954; Hesseling 1923; Vercoullie 1919

viets: uitgedos, uitgevat / dressed up, turned out Hesseling 1899; Snijman 1977

vlei: 'n moerassige laagte / a marsh Scholtz 1972

voetsek: gaan weg! / scram! Du Plooy 1966; Schuchardt 1885; Smith 1921-22

volstruis: 'n voëlsoort / ostrich, a kind of bird Bosman 1916; Du Toit 1905; Smith 1913, 1921-22; Vercoullie 1919

vremd: wisselvorm van vreemd / variant of vreemd, strange Scholtz 1963, 1985

waai: gaan / to go Hesseling 1897

waster: sluitring / washer Scholtz 1972

watter: vraende voornaamwoord / what, which, interrogative pronoun Bosman 1916; Hesseling 1899, 1923; Meyer 1900; Von Wielligh 1921-22

weeblaar: 'n plantsoort / plantain, a kind of plant Smith 1913

werda: wie's daar? / who goes there? Van Oordt 1936

ystervark: 'n diersoort / porcupine, a kind of animal Brill 1914 


\section{Bibliografie / Bibliography}

Alberts, Andries. 1955. Siembamba. TVV 12(1-2): 55-6.

siembamba

Alberts, Andries. 1961. Oorsprong van siembamba. Standpunte 14(3): 62-5.

siembamba

Alberts, Andries. 1989. Siembamba. TVV 45(1): 23-5.

siembamba

Anoniem. 1908. Het woord banje. Volkstem 8 December: 4. baie

Anoniem. 1921. Die blomnaam vergeet-my-nietjie. Huisgenoot September: 178. vergeet-my-nietjie

Anoniem. 1962. Aanvulling: emma koelemma. TVV 18(1): 33. emma koelemma

Bergerson, Jeremy. 2002a. The Etymology of Afrikaans boet and English buddy, Boots. LB 91: 63-71. boet

Bergerson, Jeremy. 2002b. An Etymology of Afrikaans mos. TNTL 118: 335-41. mos

Bergerson, Jeremy. 2004. Emphatic -s in Modern Germanic. IJGLSA 9: 83-100. beghaits: 90-4; beghieks: 91-2; bejieks: 92; bejieps: 92; bejoeks: 91; bejorries: 91; betjoeks: 91; betjoins: 91; betjols: 91; betjorries: 91; keps: 88 ; spons: 88

Boerevrou. 1926. Die Boerevrou se woordelys. April-December. April nenna: 5; May doria: 5; hotom: 5; nenna: 4, 5; June bafta: 5; bobotie: 5; kerbollie: 5; molskyn: 5; August hakskeenpleister: 4; hotom: 6; maaidekaai: 5; October mannewaar: 5; norring: 5; December Kardoes: 63

Bokhorst, M. 1940. Die woord dopper. HS 2: 71-5. Dopper

Boshoff, S.P.E. 1917. Siembamba. Brandwag 25 March: 326. siembamba

Boshoff, S.P.E. 1923-24. Oor die afleiding van g(r)amadoelas of kamadoelas. TWK 2: 15-23. gramadoelas

Boshoff, S.P.E. 1926. Review: D.F. Malherbe, Afrikaanse spreekwoorde en verwante vorme. TWK 5: 2832. dophou: $30-1$; inspan 31-2

Boshoff, S.P.E. 1927-28. Boustowwe vir die geskiedenis van Afrikaans. Latrobe (1816). TWK 6: $183-$ 94. geitjie: 191-2; graatjie: 192

Boshoff, S.P.E. 1941. Review: J. du P. Scholtz, Uit die geskiedenis van die naamgewing aan plante en diere in Afrikaans. Huisgenoot 6 June: 33, 35, 63. biskop: 33, 35; kafferkoring: 35; ratel: 35

Boshoff, S.P.E. 1950. Abiekwasgeelhout. 'n Etimologiese bydrae. TWK (NR) 10(1): 89-91. abiekwasgeelhout

Boshoff, S.P.E. 1951a. Divagasies oor dolosse. TWK (NR) 11(1): 81-94. dolos

Boshoff, S.P.E. 1951b. Kiaat en teak. TWK (NR) 11(1): 95-8. kiaat; teak

Boshoff, S.P.E. 1952. Ajoos. TWK (NR) 12(1): 102-3. ajoos; josie: 102 
Bosman, D.B. 1916. Afrikaans en Maleis-Portugees. Groningen: P. Noordhoff.

alla: 49-50; amper: 50; arrie: 50; assegaai: 50-1; atjar: 51; baadjie: 51; baar: 51-2; baklei: 52-3; baljaar: 53; basaar: 53-4; basta: 81; boet: 54-5; bottel: 55; goiing: 56; haai: 56; josie: 56-7; kaaiman: 57; kabaai: 57; kapater: 57-8; kardoes: 58; katel: 58; kiepersol: 58-9; klapper: 59; koedoe: 59; koejawel: 60; Koelie: 60; koert: 60; kraal: 61; kriek: 61; lemoen: 61-2; lukwart: 62; ma: 75-6; mandoor: 62; maskie: 62; mielie: 62-3; moveer: 63; ons: 101; oorlams: 63-4; pa: 75-6; paai: 64-5; piering: 65; pondok: 65-6; rabbedoe: 66; rissie: 66-7; seun: 73-4; sies: 67; soebat: 68; spens: 55-6; ta: 68-9; talie: 69; tjap: 69; trassie: 69-70; vieruurtjie: 70; volstruis: $70-1$; watter: $101-2$

Bosman, D.B. 1922-23. Oor die afleiding van bottel. TWK 1: 67-71. bottel

Bosman, D.B. 1937. 'n Ondersoek na die gevelariseerde -ing in Afrikaans. NT 31: 58-70. blatjang: 64; kaiings: 69; rottang: 64; toiings: 69

Botha, J.P. 1976. Die prenominale, unieke ou. FS vdMerwe: 21-5. ou

Bouman, A.C. 1922. Review: S.P.E. Boshoff, Volk en taal van Suid-Afrika. Huisgenoot March 1922: 46970 .

blaar: 469; sambreel: 469 ; stoof: 469

Bouman, A.C. 1924-25. Afrikaans ons naas Nederlands wij. TWK 3: 161-2. ons

Bouman, A.C. 1925-26. Review: D.F. Malherbe, Afrikaanse spreekwoorde en verwante vorme. TWK 4: 92-8. aardig: 95; blus: 95-6; kalant: 97

Bouman, A.C. 1928. Ontlening en relikten in Afrikaans. NT 22: 37-41. doodluiters: 40 ; tier: 41

Bouman, A.C. 1951. Het voorvoegsel ka-. TNTL 68: 97-128. kaboedel: 115

Brill, J. 1914. Die IJstervark. Over een Afrikaanse diernaam en een Oud-Grieks volksgeloof. Brandwag 1 June: $1-2,4$. ystervark

Cilliers, C.A. 1908. Het woord baing. Volkstem 15 December: 10. baie

Cilliers, C.A. 1909. 't Woord banje. Volkstem 9 February: 4. baie

Coetzee, Abel. 1944. Strandjut. TVV 1(2): 51-2. strandjut

Coetzee, Abel. 1954. Van eensgesind tot dwarstrekkerig. TVV 11(2): 1-5. eenspaaierig

Coetzee, Abel. 1961. Emma koelemma. TVV 17(2): 28-31. emma koelemma; maaifoedie: 28

Coetzee, Abel. 1964. Die es. TVV 20(3): 33-7. es

Coetzee, Abel. 1965. Die marmotjie-kaart. TO: 89-100. marmotiie

Coetzee, Abel. 1968. 'n Etimologiese woordeboek vir Afrikaans. Review: Boshoff and Nienaber, Afrikaanse etimologië. TVV 24(1): 32-6.

babbeljoentjie: 34; bolmakiesie: 34; busseltjie: 35; fluit: 35; ghwarrie: 35; kalfater: 35; koernappers: 36; snaaks: 35 
Coetzee, Abel. 1969. Dinges. TVV 25(1): 1-8. poelpetaat: 1 ; tarentaal: 1

Coetzee, A.J. 1933. Die wonderbaarlike swerftogte van kabaai. Vaderland 1 December: 13. kabaai

Coetzee, Anna E. 1983. 'n Vreemde prefiks. FS Nienaber: 95-8. kafoefel

Coetzee, Anna E. 1995. Kaboems, kabolder, kerjakker, karbonkel, karfoefel: vanwaar die hele kaboedel? SATT Supp 28: 27-44. kaboedel: 33-4; kaduks: 32; kafoefel: 34-8; karbonkel: 32-3; karjol: 32; kerjakker: 32; kerjoel: 32

Conradie, C.J. 1994. Van hoeveel tot houding: oor die subjektivering van enkele Afrikaanse kwantore. FS Raidt: 49-61. bietjie: 50-2; bra: 52-3; glad: 53-4; hoeka: 55-6

Conradie, C.J. 1995. Die partikel mos: 'n semantiese verkenning. SATT Supp 28: 45-55. mos

D'Arbez. 1916. 'n Historiese verklaring van 'n Afrikaans woord. Brandwag 1 August: 96. amper

De Bruin, P.S. 1978. Die sambreel van die kiepersol. TVV 34(2): 7-8. kiepersol

Den Besten, Hans. 2004. The Pidgin (and English) Roots of Afrikaans booi and Boesman. FS vSchalkwyk: 183-94 Boesman: 188-90; booi: 184-88; Doesman: 184

De Tollenaere, F. 1957. Review: J.L.M. Franken, Tentatiewe etimologieë. TNTL 75: 157-9. bokman: 158; haasbek: 158; keil: 158; otjie: 159; pamperlang: 158; potjierol: 159; rooms: 159

De Villiers, Meyer. 1961. Oor afrokkel en aftroggel. TGw 1: 214-6. afrokkel

De Villiers, Meyer. 1969. Die herkoms van karkatjie. TGw 9: 81-3. karkatjie

De Villiers, Meyer. 1974. Grepe uit die Afrikaanse woordeskat. FS Kempen: 31-7. sieal: 37

Du Plooy, Floris D. 1966. Einige Beobachtungen zu niederdeutschen Einschlägen im Afrikaansen. Acta Germanica 1: 159-66.

baie: 166; breekspul: 161; dalk: 161; doodluiters: 161-2; gebodder: 162; hake-krukke: 162; japsnoet: 162-3; katools: 163; kattejag: 163; kekkelbek: 163; kerjakker: 164; lekseltjie: 164; skinder: 161; skoot: 165; voetsek: 165

Du Toit, Heinrich S. 1932. Oorsprong van die woord dolos. Huisgenoot 15 April: 39. dolos

Du Toit, P.J. 1905. Afrikaansche studies. Ghent: A. Siffer.

alla: 40; assegaai: 40-1; boet: 41-3; bottel: 43-4; doepa: 44-5; doerias: 45; dogter: 69-70; dolos: 22-3; froetang: 47; geitjie: 22; goiing: 47-8; haai: 48; haai-hoei: 48; jamboes: 49; kaaiman: 49; kapok: 70-1; kiepersol: 49-50; klapper: 50; koedoe: 51; koejawel: 51-2; kool: 52; kriek: 52-3; lieplapper: 53; moesoek: 54; nonna: 54-5; paaiboelie: 55; paljas: 56; piekel: 56; pienang: 56-7; sammajoa: 57; sies: 57; sosatie: 57-8; ta: 58-9; tasal: 59; tet: 59; tjalie: 59-60; toering: 60; trassie: 60-2; vieruurtjie: 45; volstruis: 46-7

Du Toit, S.J. 1926-27. Die woord rondawel. TWK 5: 127-8.

rondawel

Du Toit, S.J. 1958a. Die malles en die dolles. Huisgenoot 4 April: 53. maltrap

Du Toit, S.J. 1958b. Ghantang, die vlugvoetige. Huisgenoot 2 May: 59. ghantang 
Du Toit, S.J. 1958c. Dis alles doppe. Huisgenoot 8 August: 69.

dop

Du Toit, S.J. 1959a. Wat sê Horak? Huisgenoot 10 April: 35. Horak

Du Toit, S.J. 1959b. Is 'n dolos dol? TVV 15(2): 9-15. dolos

Du Toit, S.J. 1959c. Dop, Dopper en Gatjieponner. TVV 15(3): 1-14. Dopper; Gatjieponner: 13-4

Du Toit, S.J. 1962. Kennetjie. TVV 18(2): 19-22. kennetjie

Du Toit, S.J. 1963. Meurant en die Doppers. TVV 19(3): 7-9. Dopper

Franken, J.L.M. 1927. Mededelinge en opienies oor Afrikaans in die neëntiende eeu. Huisgenoot 1 July: $41,43,57$. nartjie: 43 n. 8

Franken, J.L.M. 1938. Review: J.H. Rademeyer, Kleurling-Afrikaans. Die taal van die Griekwas en Rehoboth-Basters. Huisgenoot 6 May: 45, 65. lyfstuk: 45

Franken, J.L.M. 1954. Review: G.S. Nienaber, Oor Afrikaans II. OEB 20: 134-6. mandoeka: 135; vieruurtjie: 135

Goossens, Jan. 1994. Hulle. FS Raidt: 62-9. hulle; julle: 62; ons: 66

Hartwig, G.L.F. 1973. Kiaat en teak. TGw 13: 138-42. kiaat; teak

Hesseling, D.C. 1897. Het Hollandsch in Zuid-Afrika. VOAT: 139-151. baljaar: 148; laai: 148; maai: 148; paai: 148; spens: 148; trawal: 148; tronk: 148; waai: 149

Hesseling, D.C. 1899. Het Afrikaansch. Bijdrage tot de geschiedenis der Nederlandsche taal in Zuid-Afrika. Leyden: E.J. Brill.

aandag: 82-3; aapskeloeder: 82; abba: 80; aia: 88; akkerwanie: 88; amper: 88-9; arrie: 89; assegaai: 80; atjar: 89; baadjie: 89-90; baar: 90; baie: 90-1; baklei: 90; baljaar: 90; basaar: 91; basta: 114n; bermotsersanpeer: 81-2; biesroei: 79; bonkes: 105-6; borrie: 91; bredie: 91; broesa: 91; daardie: 139-40; die: 136-7; dolos: 80; ghoen: 80; hierdie: 139-40; hoeka: 80; jaarhonderd: 83; josie: 92-3; kabaai: 93; kamas: 82; kamma: 81; kapater: 93; karba: 93; kardoes: 93; karos: 81; kastig: 81; katel: 94; klip: 100n; koert: 94; kondee: 107; kraal: 94-5; Kretie: 83; laai: 95-6; laksel: 96; laksman: 96; lemoen: 96; lukwart: 96; ma: 111-2; maai: 96; makou: 96; makrol: 82; maskie: 97; mielie: 97; moesoek: 97; moveer: 97; nartjie: 97; nenna: 97; nôi: 97-8, 107; ons: 137-9; oorkrabbetjie: 95; oorlams: 98-9; pa: 111-2; paai: 99-100; pawieperske: 82; pikkenien: 100-1; poerbasledan: 86-7; pondok: 101; rabbedoe: 101; rappelkops: 83; rissie: 101; sambok: 102; sambreel: 102; seroet: 102; seun: 111; soebat: 102; spens: 91-2; stols: 83; swernoot: 84; tabeetjies: 107-8; talie: 102-3; tamaai: 103; ter duiwel: 84; tier: 104-5; tjap: 103; trawaat: 108; trawal: 103; tronk: 103-4; uitwiks: 84; veels geluk: 86; vergange: 87; viets: 82; watter: 140

Hesseling, D.C. 1909. 't Woord banje. Volkstem 9 February: 4.

baie

Hesseling, D.C. 1914. Nieuwe geschriften over het Afrikaans. Brandwag 15 January: 501-6. jilletjie: 501; kapater: 501; kremetart: 501; manel: 501 pikkenien: 501; rondawel: 501; seekoei: 502; spens: 501

Hesseling, D.C. 1916a. Africana II. De taalkundige opmerkingen van O.F. Mentzel. TNTL 35: 27588.

hanepoot: 276; kannie-koenie: 284; ons: 282; sielverkoper: 277-82 
Hesseling, D.C. 1916b. Een Afrikaanse dissertatie. Review: D.B. Bosman, Afrikaans en Maleis-Portugees. NT 10: 247-56. ons: 255-6; soebat: 255

Hesseling, D.C. 1919. Nog eens die als lidwoord. NT 13: 95-6. die

Hesseling, D.C. 1922a. De Afrikaners en hun taal. NT 16: 77-83. tronk: 81

Hesseling, D.C. 1922b. Africana III. Het Nederlands aan de Kaap tijdens Van Riebeeck. TNTL 41: 237-45. ons: $237-8$

Hesseling, D.C. 1923. Het Afrikaans. Bijdrage tot de geschiedenis der Nederlandsche taal in Zuid-Afrika. Leyden: E.J. Brill.

aapskeloeder: 79 ; amper: 82 ; assegaai: 75n, 82; baar: $74 n, 82$; baljaar: 83 ; basta: 97n; bermotsersanpeer: 77; biesroei: 76n; boet: 83; bonkes: 89-90; die: 116-8; gevreet: 80; ghoen: 77n; goiing: 83-4; kamas: 77; keil: 76n; kiepersol: 84, 90; koert: 84; Kretie: 79; loesing: 76n; ma: 95-6; makou: 85; makrol: 77; maskie: 47; moesoek: 85; moveer: 34, 85; nenna: 86; nôi: 86; ons: 119-21; oorlams: 86; ousanna: 76n; pa: 95-6; paai: 86; papelellekoors: 76n; pawieperske: 77; pikkenien: 86; rabbedoe: 76n; seun: 95; soebat: 87 ; strawasie: 79 ; tabeetjies: 91 ; talie: 87 ; trawaat: $91-2$; tronk: 72n, 88; vieruurtjie: 83 ; watter: $121-22$

H.G.V. 1919. Review: Dr. Okulis, Doppers. Huisgenoot January: 612 Dopper

J.B.D. 1932. Oorsprong van die woord dolos. Huisgenoot 18 March: 31, 51. dolos

Jeffreys, M.D.W. 1952. Tickey: Origin of the Word? ANEN 10: 25-27. tiekie

Kloppers, M.H.O. 1951-52. Hasie Aboel. TVV 8(3-4): 117-50. Hasie Aboel

Kloppers, M.H.O. 1952-53a. Hasie Aboel (vervolg). TVV 9(1): 26-32. Hasie Aboel

Kloppers, M.H.O. 1952-53b. Hasie Aboel (slot). TVV 9(2): 15-9. Hasie Aboel

Kloppers, M.H.O. 1953. Hasie-aboel: verantwoording. TVV 10(2): 33-4. Hasie Aboel

Kruisinga, E. 1906. De oorsprong van het Afrikaans. TEL 16: 417-39. die: 420-1; ons: 421

Kruyskamp, C. 1946. Banjer, banjerheer, banjaard. TNTL 64: 51-4. baie

Labuschagne, F.J. 1936. Review: S.P.E. Boshoff, Etimologiese woordeboek van Afrikaans. Koers 4(3): 323. dusketyd: 33; manewales: 33

Lategan, Felix V. 1987. Dolosse inspan. 'n Afrikaanse boerekinderspel. TVV 43(1): 4-14. dolos

Laubscher, F.X. 1941. Die naam kafferkoring. Huisgenoot 4 Julie: 45. kafferkoring

Lehmann, F. Rudolph. 1945. 'n Paar gedagtes oor die naam Boesman. TVV 2(1): 1-6. Boesman

Le Roux. J.J. 1919. Het lidwoord die. NT 13: 45. die 
Le Roux, J.J. 1936. Die woordjie ou. Huisgenoot 18 September: 43.

ou

Le Roux, T.H. 1957. Taaloudhede. TVV 13(3): 1-7. bafta: 5-6; liefêr: 4-5; tweealfaamsvat: 3-4

Leys, Odo. 2005. Versterkte voegwoorden in het Vlaams: lat, lof en nof. TT 57: 111-25. lat: $111-8$

Liebenberg, H.C. 1976. Matroostaal in Afrikaans. Klasgids 11(3): 5-16. afdraand: 6 ; basta: 5 ; katel: 6 ; kombers: 6 ; paai: 6 ; spens: 6 ; talie: 6

Lubbe, H.J. 1967. Opmerkings n.a.v. hy is bokveld toe. TVV 23(1-2): 20-4. bokveld

Lubbe, H.J. 1968. Opmerkinge n.a.v. enkele etimologiese afleidinge deur proff. Boshoff en Nienaber in Afrikaanse etimologieë. TVV 24(3-4): 55-8. kluitjie: 55; ransel: 58; stapelgek: 55-7; swets: 57

Lubbe, H.J. 1970. Review: Johan Combrink, Waar kom daardie woord vandaan? TVV 26(2): 28-9. hortjie: 29; skalk: 29; stryk: 29

Lubbe, Johan. 2004. Aardig in Afrikaans en Nederlands - 'n diachroniese beskrywing van die betekenisverskille. FS vSchalkwyk: 255-67. aardig

Malherbe, D.F. 1922-23. Review: S.P.E. Boshoff, Volk en taal van Suid-Afrika. TWK 1: 112-23. agterlosig: 121; baadjie: 119; bak: 115-6; blaar: 118-9; blus: 122; dolos: 122; doodluiters: 120; ewwatrewwa: 120; geit: 118; pamperlang: 122; pelloe: 119; seties: 123; sieal: 122; skalt en walt: 120-1; streepsuiker: 119 ; toiings: 119

Malherbe, D.F. 1924-25. Opmerking. TWK 2: 162 ons

Meyer, Heinrich. 1900. Der Ursprung der Burensprache. FS HG: 83-121. maskie: 108; ons: 112; smous: 90 ; watter: 89

Nienaber, G.S. 1952a. Twee Afrikaanse woorde. TWK (NR) 12(2): 18-25. Boesman: 18-23; hartbeeshuis: 23-5

Nienaber, G.S. 1952b. Herkoms van hartbeeshuisie. Vaderland Ekstra 20 September: 10-11. hartbeeshuis

Nienaber, G.S. 1953. Die duwweljoe in wees vir iemand. Vaderland Ekstra 11 April: 10-11. duwejoe

Nienaber, G.S. 1954a. 'n Aantekening by eena, deena. TVV 11(1): 15-6. eena, deena

Nienaber, G.S. 1954b. Waar kry jy daardie woord? 1. Rysmiere. Huisgenoot 9 July: 11. rysmier

Nienaber, G.S. 1954c. Waar kry jy daardie woord? 2. In die dubbeljoe. Huisgenoot 16 July: 17. duwejoe

Nienaber, G.S. 1954d. Waar kry jy daardie woord? 5. Graatjiemeerkat. Huisgenoot 6 August: 29. graatjie

Nienaber, G.S. 1954e. Waar kry jy daardie woord? 6. Hartbeeshuisie. Huisgenoot 13 August: 17. hartbeeshuis

Nienaber, G.S. 1954f. Waar kry jy daardie woord? 7. Boesman. Huisgenoot 20 August: 11. Boesman

Nienaber, G.S. 1954g. Waar kry jy daardie woord? 8. Hottentot. Huisgenoot 27 August: 23. Hottentot

Nienaber, G.S. 1954h. Waar kry jy daardie woord? 9. Mamparra. Huisgenoot 3 September: 35. mamparra 
Nienaber, G.S. 1954i. Waar kry jy daardie woord? 10. Hy is nog baar. Huisgenoot 10 September: 43. baar; oorlams

Nienaber, G.S. 1954j. Waar kry jy daardie woord? 11. Woorde in Bantoe bewaar. Huisgenoot 17 September: $29,31$.

lepaai

Nienaber, G.S. 1954k. Waar kry jy daardie woord? 12. Handkous en doodgooi. Huisgenoot 24 September: 27, 29.

doodgooi

Nienaber, G.S. 1954l. Waar kry jy daardie woord? 13. Kalkoene en tarentale. Huisgenoot 8 Oktober: $45,47$.

kalkoen: 45 ; tarentaal: 45, 47

Nienaber, G.S. $1954 \mathrm{~m}$. Waar kry jy daardie woord? 15. Bolmakiesie slaan. Huisgenoot 29 October: 12-3.

bolmakiesie

Nienaber, G.S. 1954n. Waar kry jy daardie woord? 16. Biltong. Huisgenoot 5 November: 40-1. biltong

Nienaber, G.S. 1956a. Oor die herkoms van die woord Hottentot. Standpunte 10(5): 28-46. Hottentot

Nienaber, G.S. 1956b. Oor die herkoms van die woord Hottentot (vervolg). Standpunte 10(6): 44-55. Hottentot

Nienaber, G.S. 1965. Die bok in drabok. Taalfasette 1: 54-9. drabok

Nienaber, G.S. 1966. Die wyn in aalwyn. FS Louw: 108-17. aalwyn

Nienaber, G.S. 1968a. Rondawel. TVV 24(3-4): 1-10. aalwyn: 8; drabok: 8; resnawel: 10; rondawel

Nienaber, G.S. 1968b. Wat sê Horak? Standpunte 21(5): 31-6. Horak

Nienaber, G.S. 1989. Sonde met hartbees in hartbeeshuis. TGw 29: 180-92. hartbeeshuis

Nienaber, G.S. 1991. Is die modale bywoord rêrig rêrig Afrikaans? TGw 31: 128-40. rêrig

Nienaber, G.S. 1994a. Aantekeninge by fieterjasies. TGw 34: 64-6. fieterjasies

Nienaber, G.S. 1994b. Kanttekening: Makrappa. TGw 34: 66. makrappa

Nienaber, G.S. 1995. Die etimologie van dolos. SATT Supp 23. dolos; makrappa: 58-9; mamparra: 58

Odendal, F.F. 1964. Karet en botel. TVV 20(2): 50-3. botel: 52-3; karet: 50-2

Odendal, F.F. 1966. Kierang en koerang. TGw 6: 151-4. froetang: 152n; kierang; koerang

Odendal, F.F. 1994. Waar die kranse antwoord gee. FS Raidt: 29-32. krans

P. 1911. Het Afrikaanse woord ou. Goede Hoop November: 393-4. ou

Paardekooper, P.C. 1969. Een Fransvlaams-Zeeuwse parallel: ons 'wij'. TT 21: 211-5. ons: 215 
Paardekooper, P.C. 1990. Een voegwoordraadsel: Lat 'dat'. SATT 8: 174-7. lat

Pauwels, J.L. 1959a. Afrikaans hierdie, daardie. LB Bijblad 48: 1-3. daardie; hierdie

Pauwels, J.L. 1959b. FS Pauwels: 51-70. oes: 63

Pienaar, P. de V. 1928-29. Kannie-koenie of koenie-kannie (ghoenie-ghannie, ens.). TWK 10: 99-101. kannie-koenie

Rautenbach, W.P. 1978. Die verlies van die woord tof in Afrikaans. TVV 34(3): 20-3. tof

Roberge, Paul T. 2001. Diachronic Notes on the Afrikaans Demonstrative Pronouns. FS vRensburg: 124-36. daardie; hierdie

Roberge, Paul T. 2002. The Modal Elements mos and maskie in Cape Dutch. LS 24: 397-408. maskie; mos

Scholtz, J. du P. 1963. Taalhistoriese opstelle. Voorstudies tot 'n geskiedenis van Afrikaans. Pretoria: J.L. van Schaik. baie: 210-1(n), 213: bleik: 246; brei: 182; daardie: 138-9; dalk: 186; die: 134-8; goël: 193-4; hekel: 234; hierdie: 138-9; hom: 52-3; hulle: 61, 62, 64, 96-101, 105, 111-5; julle: 59-64, 82-86; kaiings: 171, 209; karet: 250; kasarm: 240-1; kês: 238-9; koël: 234; lawaai: 219; maaier: 171; moeg: 247; moggel: 191; motjie: 222; nôi: 211n; nuut: 245; oggend: 191; ons: 93-6, 100-1, 111-2, 115-6; portfesiedeur: 222; ramkie: $211 \mathrm{n}$; rêrig: 151; resep: 219; rottang: 211(n); se: 52, 119-21; sens: 247; soms: 219-20; sooibrand: 172; sproei/spru: 242; sulle: 60-4, 96-8, 113; tamaai: 211n; teiken: 246; toiings: 209; u: 64-7, 74-7; vremd: $246-7$

Scholtz, J. du P. 1972. Afrikaans-Hollands in die agtiende eeu. Verdere voorstudies tot 'n geskiedenis van Afrikaans. Cape Town: Nasou.

aalwyn: 96; Afrikaner: 95; als: 102; askuus: 91-2; aspres: 91-2; astrak: 91-2; astrant: 94; baklei: 82-3; blaasbalk: 102; daardie: 40; dalk: 77; die: 35-9; duwejoe: 50; els: 102; gunter: 102; hê: 55; het: 10-1; hierdie: 40; hom: 79; kiewiet: 46; koejawel: 98; likkewaan: 96; manewales: 96; mos: 79; olienhout: 48; ontwil: 79; oond: 48, 98; oop: 78; pikkewyn: 96; prinjeel: 96; rasper: 88; rinneweer: 96; rottang: 79; sarlot: 97; sens: 102; skinder: 70; stawel: 50; swael: 48; swetterjoel: 96; tamboekie: 81; tameletjie: 78, 97; tarentaal: 67; tjôwa: 50; twak: 50, 54, 83; vark: 78n; vlei: 83; waster: 94, 95

Scholtz, J. du P. 1979. Boerneef se taal. Burger 30 May: 12. bokrin; bokruit; nimlik

Scholtz, J. du P. 1985. Vroeë taalkundige studies. TGw 25: 80-105. ag: 101; akkerboom: 94, 100; burg: 103; het: 97; karet: 102; vremd: 93

Schoonees, P.C. 1950. Aantekening oor die woord ongans. TWK (NR) 10: 92. ongans

Schuchardt, Hugo. 1885. Review: N. Mansvelt, Proeve van een Kaapsch-Hollandsch idioticon. Literaturblatt für germanische und romanische Philologie 11: 464-70.

aapskeloeder: 466; arrie: 468; balie: 468; boeglam: 466; katel: 468-9; maskie: 468; ons: 468; poerbasledan: 468; se: 466; veels geluk: 467; voetsek: 469

Schuchardt, Hugo. 1891. Kreolische Studien IX: Über das Malaioportugiesische von Batavia und Tugu. Sitzungsgeschichte der Kaiserlichen Akademie der Wissenschaften in Wien, Philosophisch-historische Classe 122(12): 1-256.

baar: 17, 237; katel: 119n; nôi: 250; oorkrabbetjie: 127n; oorlams: 16-7; rabbedoe: 108n

Schuhmacher, W.W. 1973. Once more ons. Standpunte 28(8): 60.

ons 
Smith, J.J. 1913. Review: C. Pettman, Africanderisms. Brandwag 1 December: 394-95, 397-401. alikreukel: 398; alla: 399; arrie: 400; borrie: 400; bredie: 400; geilsiekte: 398; gus: 398; harslag: 399; kalossies: 399; kaparring: 400; kapater: 400; kardoes: 400; kiaat: 400; koedoe: 400; koejawel: 400; koggelman: 400; makou: 400; manel: 399; mielie: 400; mombakkies: 399; moveer: 400; nooi: 399; ons: 397n; platanna: 399; portfesiedeur: 399; ramkie: 400; rissie: 400; sa: 399; seekoei: 399; skaai: 399; swernoot: 399; tamaai: 399; tasal: 400; volstruis: 400; weeblaar: 399

Smith, J.J. 1921-22. Notes on G.R. von Wielligh's Ons geselstaal. Huisgenoot January 1921-July 1922. January '21 bottel: 393 n. 15; lemoen: 393 n. 14; kapel: 392 n. 7; nôi: 393 n. 12; perske: 392 n. 5; tarentaal: 393 n. 13; tiekie: 393 n. 20; volstruis: 392 n. 4; February '21 pikkenien: 419 n. 5; March '21 ghoen: 457 n. 2; meestergoed: 458 n. 8; April '21 kiets: 497 n. 5; June '21 geitjie: 63 n. 2; July '21 nôi: 99 n. 8; papkuil: 98 n. 1; ramkie: 99 n. 8; tamaai: 99 n. 8; August '21 duiwelsterk: 146 n. 3; gramadoelas: 145 n. 2; paaiboelie: 145 n. 3; perske: 146 n. 1; seekoei: 145 n. 1; voetsek: 146 n. 8; March '22 gramadoelas: 452 n. 1; July '22 filekommis: 113 n. 2

Smith, J.J. 1938. Oorlam en baar. Stellenbossche Oudstudent 8(1): 18-23. baar, oorlams

Smith, J.J. 1942. 'n Bietjie oor baie. TO: 173-80. baie

Snijman, F.J. 1977. Nederlandse lexicografische baanbrekers in Zuid-Afrika. FS Kruyskamp: 189202. dam: 194; hakskeen: 192; oes: 193; vaderlands: 193-4; viets: 192

Taalman. 1946. Op taalbesoek by Boerneef. OEB 12: 64-68; 143-147. bokrin: 66; bokruit: 66; skaaprin: 66; skaapruit: 66

Te Winkel, J. 1896. Het Nederlandsch in Noord-Amerika en Zuid-Afrika. VOAT: 113-134. alikreukel: 115-116; amper: 121; assegaai: 124; baadjie: 121; baar: 121; baklei: 122; baljaar: 122; koerang: 123; kras: 121; mielie: 123; oorlams: 121-122; sambok: 123-124; sambreel: 117; soebat: 122

Valkhoff, Marius F. 1955. Beskouings oor woordontlening II. TVV 12(1-2): 21-9. bermotsersanpeer: 28n-29n; dolos: 23; kastrol: 23; lumier: 23; moveer: 27; pikkenien: 27; tronk: 27

Valkhoff, Marius F. 1969. Review: Boshoff and Nienaber, Afrikaanse etimologië. AS 28: 143-6. baie: 145; baljaar: 146; basta: 146; nôi: 146; piering: 146; pikkenien: 145; tamaai: 146; tronk: 146

Van Blerk, N. 1958. Oesbeentjie. Huisgenoot 28 July: 53. oesbeentiie

Van den Berg, B. 1965. Heeft het Afrikaans zich uit het Hollands ontwikkeld? FS Scholtz: 25-38. die: $32-7$

Van der Meulen, R. 1917. Robbedoes. TNTL 36: 1-9. rabbedoe

Van Ginneken, J. 1927. De twee beteekenissen van kuieren. TNTL 46: 188-9. kuier

Van Oordt, J.F. 1916. Review: D.B. Bosman, Afrikaans en Maleis-Portugees. Brandwag 1 October: 1423, 145-9. amper: 147; assegaai: 147; baie: 145n; basaar: 147; goiing: 147; josie: 147; kapater: 147; katel: 147; koedoe: 147; moveer: 147; oorlams: 147; piering: 148; pondok: 148; rissie: 148; soebat: 148

Van Oordt, L.C. 1936. Grepe uit die gesproke taal van 1700. Huisgenoot 15 May: 27. booi, loesing, maskie, ons, sa, werda

Van Oordt, L.C. 1941. Boesman alias Bosjesman. Huisgenoot 14 March: 27, 69. Boesman

Van Rensburg, Christo. 1996. Is Koi-invloed op Afrikaans werklik Koi-invloed? TT Themanummer 9: 128-45. ons: 137-41 
Venter, Hertzog. 1968. Review: Boshoff and Nienaber, Afrikaanse etimologië.. Standpunte 21(5): 45-7. ghwarrie: 47 ; gus: 47 ; nè: 47

Vercoullie, J. 1919. Negerhollands molee, Afrikaans boetie, katjipiering, bibies, bottel, Ou Sanna, ewwatrewwa, foolstruis. TNTL 38: 302-6.

biebies: 305; boet: 304; bottel: 305; ewwa-trewwa: 305; katjiepiering: 304; ousanna: 305; vieruurtjie: 305; volstruis: 306

Von Wielligh, G.R. 1921-22. Ons geselstaal. Huisgenoot January 1921-July 1922.

March '21 pasella: 457; July '21 baie: 98-9; papkuil: 98; August '21 agostou: 145-6; beesbyter: 145; eendjies: 145; gansies: 145; kankerbossie: 145; makoppa: 145; perske: 146; seekoei: 145; watter: 146; September '21. herneuters: 200

Vor der Hake, J.A. 1911. Is de beleefdheidsvorm $U$ 'n verbastering van $U E d$. ? NT 5: 16-24. hulle: $19 ;$ ons: $19 ; u$

Waher, Hester. 2001. Woorde wat wegraak: oorgeërfde Franse leenwoorde in Afrikaans. FS vRensburg: 157-63. menteneer: 157-8; resnawel: 158-9; spandabel: 161-2

Walton, James. 1987. Hartbeeshuis and hartbeeshut. TVV 43(1): 15-23. hartbeeshuis

\section{Lys Afkortings / List of Abbreviations}

Acta Germanica = Acta Germanica. Zur Sprache und Dichtung Deutschlands, Österreichs und der Schweiz (Cape Town)

ANEN = Africana Notes and News (Johannesburg)

$A S=$ African Studies (Johannesburg)

Brandwag = Die Brandwag (Pretoria)

Burger $=$ Die Burger $($ Cape Town $)$

FS HG = Festschrift dem Hansischen Geschichtsverein und dem Verein für niederdeutsche Sprachforschung dargebracht zu ihrer Jahresversammlung in Göttingen Pfingsten 1900. Göttingen: Dieterichsche Univ-Buchdruckerei von W. Fr. Kästner.

FS Louw = Smal swaard en blink. Bundel aangebied aan N.P. van Wyk Louw by geleentheid van sy sestigste verjaardag, 11 Junie 1966. Pretoria/Cape Town: Academica.

FS Kempen = Taalkunde - ' $n$ Lewe. Studies opgedra aan Prof. Dr. W. Kempen by geleentheid van sy 65ste verjaardag, 3 Julie 1974. Odendal, F.F., D.C. Hauptfleisch, J.E. Loubser (Eds.). Cape Town/Johannesburg: Tafelberg.

FS Kruyskamp $=$ Opstellen door vrienden en vakgenoten aangeboden aan Dr. C.H.A. Kruyskamp ter gelegenheid van zijn 65ste verjaardag en van zijn afscheid als redacteur van het Woordenboek der Nederlandsche Taal en als enige bewerker van Van Dale. Heestermans, Hans (Ed.). The Hague: Martinus Nijhoff.

FS Nienaber $=$ G.S. Nienaber - ' $n$ huldeblyk. Studies opgedra aan Prof. Dr. G.S. Nienaber in sy tagtigse jaar. Sinclair, A.J.L. (Ed.). 1983. Bellville: University of the Western Cape.

FS Pauwels = Verzamelde Opstellen. Aangeboden aan de Auteur ter Gelegenheid van zijn 65e Verjaardag. Engels, L.K., J. van Haver, G.Geerts (Eds.). 1965. Assen: Van Greunen.

FS Raidt = Nuwe perspektiewe op die geskiedenis van Afrikaans: opgedra aan Edith H. Raidt. Olivier, Gerrit, Anna Coetzee (Eds.). 1994. Johannesburg: Southern Book Publishers.

FS Scholtz $=$ Dietse studies. Bundel aangebied aan Prof. Dr. J. du P. Scholtz by geleentheid van sy vyf-ensestigste verjaardag 14 Mei 1965. Lindenberg, E., Edith Raidt, J.A. Verhage (Eds.). Cape Town/Pretoria: Academica. 
FS vdMerwe = Gedenkbundel H.J.J.M. van der Merwe. De Klerk, N.J., F.A. Ponelis (Eds.). 1976. Pretoria: J.L. van Schaik.

FS vRensburg = Taallandskap. Huldigingsbundel vir Christo van Rensburg. Carstens, Adelia, Heinrich Grebe (Eds.). 2001. Pretoria: Van Schaik.

FS vSchalkwyk = 'n Man wat beur. Huldigingsbundel vir Dirk van Schalkwyk. Botha, Willem (Ed.). 2003. Stellenbosch: Bureau of the WAT.

Goede Hoop = De Goede Hoop (Cape Town)

Handhaaf = Handhaaf (Johannesburg)

HS = Historiese Studies (Pretoria)

Huisgenoot $=$ Die Huisgenoot (Cape Town)

IJGLSA = Interdisciplinary Journal for Germanic Linguistics and Semiotic Analysis (Berkeley)

Koers $=$ Koers. Tweemaandelikse tydskrif (Potchefstroom)

$L B=$ Leuvense Bijdragen (Louvain)

$L S=$ Language Sciences (Oxford)

$N T=$ De Nieuwe Taalgids (Groningen)

OEB = Ons Eie Boek (Cape Town)

Raidt 1994 = Raidt, Edith H. 1994. Historiese taalkunde. Studies oor die geskiedenis van Afrikaans. Johannesburg: Witwatersrand University Press.

SATT = Suid-Afrikaanse Tydskrif vir Taalkunde/South African Journal of Linguistics (Pretoria)

SATT Supp $=$ Suid-Afrikaanse Tydskrif vir Taalkunde/South African Journal of Linguistics. Supplement/Supplement (Pretoria)

Standpunte $=$ Standpunte $($ Cape Town)

Taalfasette $=$ Taalfasette $($ Pretoria $)$

$T G w=$ Tydskrif vir Geesteswetenskappe (Pretoria)

$T E L=$ Taal en Letteren (Zwolle)

TNTL $=$ Tijdschrift voor Nederlandse Taal- en Letterkunde (Leyden)

TO = Taalkundige opstelle. P.J. Nienaber (Ed.). 1965. Cape Town/Amsterdam: A.A. Balkema.

TT = Taal en Tongval (Amsterdam/Ghent)

$T V V=$ Tydskrif vir Volkskunde en Volkstaal (Johannesburg)

TWK $=$ Tydskrif vir Wetenskap en Kuns (Pretoria)

TWK $($ NR) $=$ Tydskrif vir Wetenskap en Kuns (Nuwe Reeks) (Pretoria)

Vaderland = Die Vaderland (Johannesburg)

VOAT = Vroeë opvattings oor Afrikaans as taal. G.S. Nienaber (Ed.). 1984. Johannesburg/Cape Town: Perskor.

Volkstem $=$ De Volkstem $($ Pretoria) 